\title{
Activités
}

6-1 | avril 2009

Varia

\section{La résolution de problèmes professionnels sur simulateur}

Professional problem solving using a simulator

La resolución de problemas profesionales en simulador

Pierre Pastré, Pierre Parage, Jean-François Richard, Emmanuel Sander, Jean-Marc Labat et Michel Futtersack

\section{CpenEdition}

\section{Journals}

Édition électronique

URL : http://journals.openedition.org/activites/2122

DOI : 10.4000/activites. 2122

ISSN : $1765-2723$

Éditeur

ARPACT - Association Recherches et Pratiques sur les ACTivités

Référence électronique

Pierre Pastré, Pierre Parage, Jean-François Richard, Emmanuel Sander, Jean-Marc Labat et Michel Futtersack, "La résolution de problèmes professionnels sur simulateur », Activités [En ligne], 6-1 | avril 2009, mis en ligne le 15 avril 2009, consulté le 23 avril 2019. URL : http://journals.openedition.org/ activites/2122; DOI : 10.4000/activites. 2122

\section{c) (i) $९$}

Activités est mis à disposition selon les termes de la licence Creative Commons Attribution - Pas d'Utilisation Commerciale - Pas de Modification 4.0 International. 


\title{
La résolution de problèmes professionnels sur simulateur $^{1}$
}

\section{Pierre Pastré}

Chaire de communication didactique

\section{Pierre Parage}

Chaire de formation des adultes, CNAM

\section{Jean-François Richard, Emmanuel Sander}

Laboratoire Paragraphe, équipe CRAC, Paris 8

\section{Jean-Marc Labat}

Lip6, Paris 6

\section{Michel Futtersack}

Faculté de Droit, Paris 5

\begin{abstract}
Professional problem solving using a simulator.

This paper stems from research on professional problem solving, conducted by a team of researchers from three different areas: cognitive psychology, artificial intelligence, and professional didactics. We used the material collected from 1992 to 1995 by Pierre Pastré concerned with the activity of setters correcting defects on plastic objects produced by an injection press; this professional problem solving was performed on a simulator. The goal of the research was to model the setters' activity using two approaches: an approach based on constraints (Richard) and another on knowledge (Labat \& alii). The results of these modeling frameworks allowed for a more detailed analysis of the strategies mobilized by the setters. It is this analysis of the strategies of setters, within their organization and as part of their evolution, that is addressed by this paper. The analysis is done from a perspective looking at the conceptualization in action. Two main results were found: most of the setters' strategies combine an empirically oriented approach (led by the defects) and a symbolic approach (led by the curve of pressures), with a development which results in increasing importance for the symbolic approach, but without it the empirical approach disappears. Despite this, , the crucial moment in the evolution of strategies occurs when setters shift from a linear type conceptualization (a defect - a cause) to a systemic type (which takes into account the compensations).
\end{abstract}

KEYWORDS

Simulation, activity modelisation, problem solving, professional didactics, conceptualization in action.

1. La recherche dont est issu ce papier a été financée par une subvention du programme de sciences cognitives TCAN du CNRS. 
La recherche présentée dans cet article porte sur la résolution de problèmes professionnels. Dans la littérature, on trouve de nombreux travaux de psychologie qui portent sur la résolution de problèmes, à partir du cadre de référence de Newell et Simon (1972). Ils ont surtout porté sur des situations de jeu ou de casse-tête (tour de Hanoï, missionnaires et cannibales, Passalong: Richard, 1999), où la difficulté ne venait pas de la complexité des connaissances à mobiliser, mais du caractère inhabituel de la situation posant problème. D'un autre côté, en psychologie du travail, l'importance des situations-problèmes a été mise en évidence depuis longtemps. Hoc (1990) par exemple a montré que les activités de diagnostic peuvent être interprétées comme des situations de résolution de problème: qu'il s'agisse de diagnostic de pannes, d'incidents, de dysfonctionnement ou simplement d'un moment critique, l'activité de diagnostic est caractérisée par le fait qu'il n'existe aucune procédure permettant avec certitude d'atteindre le but recherché. On peut d'ailleurs penser que la capacité d'un opérateur à résoudre les problèmes professionnels qu'il rencontre est un bon indicateur de son niveau de compétence (Hatchuel, \& Weil, 1992).

Ce point de vue, qui relie mobilisation des compétences et résolution de problèmes, a été développé en didactique professionnelle (Pastré, 2008), c'est-à-dire dans une perspective qui consiste à analyser le travail en vue de la formation des compétences professionnelles. C'est cette approche qui est mobilisée ici. Trois points permettent de la caractériser: 1 / Comme nous nous situons dans une perspective de formation, les compétences analysées ne sont pas les compétences directement mobilisées par les acteurs dans le cadre de leur activité professionnelle de référence, mais les compétences qu'ils sont capables de mobiliser quand ils sont confrontés à des situations critiques, voire inhabituelles, qui les obligent à se placer en situation de résolution de problèmes. C'est une différence importante entre une approche de psychologie ergonomique, qui analyse l'activité effective, et une approche de didactique professionnelle, qui, centrée sur la formation, analyse ce qu'un opérateur est capable de déployer comme activité quand il ne peut pas s'appuyer sur les procédures habituelles du métier. 2/ C'est dans ces situations critiques de résolution de problèmes professionnels qu'il est possible d'analyser l'organisation de l'activité des acteurs. Non seulement nous faisons l'hypothèse que toute activité humaine efficiente est organisée (Vergnaud, 1996), mais nous pensons également qu'au cœur de cette activité il y a de la conceptualisation, non pas au sens où les opérateurs agiraient en appliquant un plan pré-établi, mais au sens où ils disposent d'éléments conceptuels organisateurs de leur activité ( « concepts pragmatiques » ou « jugements pragmatiques », Pastré, 2008), implicites ou explicites, qui guident et orientent leur action en fondant un bon diagnostic de situation. L'analyse de l'activité va consister à identifier ces éléments conceptuels organisateurs de l'activité qui permettent de comprendre les stratégies que les acteurs sont capables de mettre en œuvre. 3/ Pour ce faire, deux moyens ont été utilisés: d'une part, nous avons travaillé sur simulateur, dans une perspective de " pédagogie des situations » (Pastré, 2004), en utilisant des situations issues du travail pour analyser compétences et stratégies. Cela a permis en outre d'observer des opérateurs différents confrontés à la même suite de problèmes. D'autre part, nous nous sommes appuyés sur le travail de modélisation de l'activité effectué par Richard et Sander, dans le cadre du modèle des contraintes (Richard, Poitrenaud, \& Tijus, 1993), ainsi que sur la modélisation réalisée par Labat et Futtersack, dans le cadre d'un modèle basé sur les connaissances (Labat, Pastré, Parage, Futtersack, Richard, \& Sander, 2007). Il est en effet important de pouvoir procéder à une modélisation de l'activité des acteurs, sous la forme d'un répertoire de règles d'action ou de connaissances, pour pouvoir en extraire les éléments conceptuels centraux, qui justifient les stratégies des acteurs et leur donnent sens.

La situation de résolution de problème qui sert de support à notre recherche est la suivante: il s'agit, pour des régleurs de presses à injecter en plasturgie, de corriger des défauts qui apparaissent sur les produits fabriqués. Comme nous l'avons indiqué, le travail d'analyse n'a pas porté sur la situation professionnelle de référence, mais sur une simulation, en profitant du fait que la situation professionnelle était suffisamment maîtrisée pour qu'elle puisse être modélisée sans distorsions trop importantes. Le simulateur réalisé est un simulateur de résolution de problème (Pastré, 2005): il ne cherche pas à reproduire intégralement le procès de travail, comme dans les simulateurs " pleine échelle », mais il se concentre sur la mise en scène du problème issu de la situation professionnelle de réfé- 
rence, qui permet de donner la mesure du niveau de compétence mobilisable par les acteurs: «On simule non pas le réel, mais le problème » (Samurçay, \& Rogalski, 1992).

Cette recherche a eu une forte dimension interdisciplinaire,elle a mobilisé trois équipes: l'une en psychologie cognitive (Richard, Sander); la deuxième en intelligence artificielle (Labat, Futtersack); la troisième en didactique professionnelle (Pastré, Parage). Le but initial fut d'utiliser les données recueillies dans deux recherches antérieures (Pastré, 1994), pour tester différentes méthodes de modélisation d'une activité de résolution de problèmes: méthode basée sur les contraintes (Richard et al.); méthode basée sur les connaissances (Labat et al.). Deux articles, parus (Labat, Pastré, Parage, Futtersack, Richard, \& Sander, 2007) ou à paraître (Richard, Pastré, Parage, Sander, Futtersack, \& Labat J, à paraître), présentent ces deux types d'analyse. Le présent article a pour objectif de montrer que la démarche générale qui a présidé à la recherche produit des analyses en retour dans le sens inverse: il cherche à montrer comment une modélisation de l'activité de résolution de problèmes des opérateurs permet de mieux comprendre sur quelle forme de conceptualisation reposent leurs stratégies.

Nous procéderons en quatre parties: 1/ La première partie consiste à présenter la situation professionnelle de référence, sa modélisation, le corpus recueilli et son codage. 2/ La deuxième partie empruntera une démarche historique: la recherche a connu trois étapes, et il nous paraît intéressant de décrire ces étapes, pour voir comment l'analyse des stratégies a évolué. 3/ La troisième partie constituera un retour sur la question des stratégies des acteurs, en montrant notamment comment s'est noué l'échange entre le point de vue de la psychologie ergonomique et celui de la modélisation de l'activité. 4/ La quatrième partie constituera une discussion.

\section{1.- La situation professionnelle de référence}

\section{1.- La machine et le travail des régleurs}

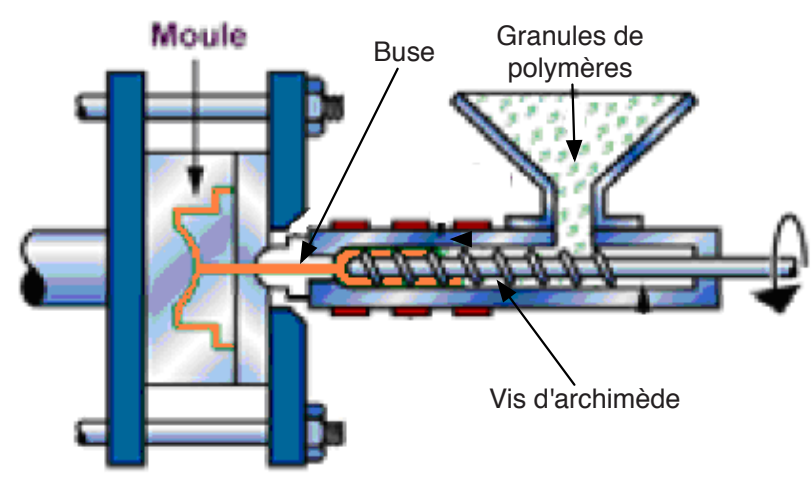

Figure 1: schéma d'une presse à injecter

Figure 1: diagram of an injection machine

La tâche choisie pour servir à l'analyse de résolutions de problèmes en situation professionnelle est une tâche de réglage de presses à injecter à commande numérique. Les caractéristiques de la tâche sont les suivantes:

1 - Une presse à injecter est une machine qui transforme des granulés plastiques en objets moulés ayant une forme définie. La technique de l'injection plastique s'apparente au travail de fonderie: on chauffe une quantité précise de matière plastique jusqu'à son point de fusion. On l'injecte alors sous forte pression dans les empreintes d'un moule, où elle est refroidie jusqu'à solidification. Il y a deux phases dans l'injection, une phase dynamique (l'injection proprement 
dite) et une phase statique (le maintien pendant la solidification). Pendant la phase de maintien, une pression de maintien est appliquée sur la matière injectée pour compenser le retrait de matière consécutif à la solidification.

2-Les opérateurs qui ont été observés sont des régleurs: leur tâche consiste principalement à intervenir en cas d'apparition de défauts sur les produits pour les corriger. Ces défauts sont assez nombreux et fréquents. Ils peuvent apparaître en phase dynamique comme en phase statique. Les défauts peuvent avoir plusieurs origines: défauts de matière plastique, défauts de réglage de la machine, usure des pièces de celle-ci. Notre analyse a porté sur la correction de défauts dus au réglage et partiellement sur la correction de défauts dus à l'usure de pièces, dans la mesure où cette usure avait un impact sur les réglages.

3-Les machines qui ont servi de support à l'analyse de la tâche sont des presses à injecter à commande numérique, de type MOCN (machine-outil à commande numérique). Une presse à injecter n'est pas un système technique dynamique, au sens donné par Hoc (1990): il n'y a pas (ou très peu) de dynamique propre au système. Les transformations résultent de l'action des opérateurs. On peut donc traiter les corrections de défauts comme un cas particulier de résolution de problèmes par transformation d'états: un état initial (la présence d'un défaut) entraîne un nombre $\mathrm{N}$ (N pouvant être égal à 1$)$ d'opérations de la part du régleur, jusqu'à obtention d'un état final (absence de défaut).

\section{2.- Analyse de la tâche de réglage}

Il y a dans le cycle de fonctionnement de la machine un point remarquable, le point de commutation, qui marque le passage entre la phase dynamique d'injection, caractérisée par une vitesse et une pression, et la phase statique, ou phase de maintien, caractérisée par un arrêt du mouvement de la machine, qui traduit un équilibre entre la pression exercée par la machine (pression de maintien) et la pression en retour de la matière. Une machine bien réglée est celle où on passe de l'injection au maintien au moment précis où les empreintes du moule sont pleines. On peut donc écrire une égalité, qui dans la pratique correspond à un équilibre: une presse est bien réglée quand le volume de matière dosée correspondant à la course de dosage ${ }^{2}$ est égal au volume que les empreintes du moule peuvent contenir. Cet état d'équilibre a reçu dans les ateliers le nom de «bourrage ». Nous le qualifierons de « concept pragmatique » (Pastré, 1994): il permet de distinguer les deux régimes de base de fonctionnement de la machine, le régime normal (quand il y a équilibre entre la pression machine et la pression matière au moment de la commutation) et le régime compensé (quand la pression machine est supérieure à la pression matière au moment de la commutation, ce qui entraîne un deuxième remplissage pendant la phase de maintien). Le concept de bourrage est donc essentiel pour établir un diagnostic de régime de fonctionnement de la machine.

Mais le bourrage en plasturgie s'accompagne de transformations thermiques, donc de changements d'états de la matière. Il faut également contrôler ces dimensions de l'action, d'où une deuxième égalité : pendant la phase de maintien, pendant laquelle le plastique se cristallise en se refroidissant, il faut que la diminution de son volume soit compensée par la pression de maintien qui va «nourrir » les pièces jusqu'à ce qu'elles soient solidifiées. Le retrait de matière pendant la solidification est ainsi le deuxième concept organisateur qui va guider l'action des régleurs. Bourrage et retrait, les deux concepts organisateurs, forment un couple: pour que le réglage de la machine soit correct, il faut que, pendant la phase statique de maintien, le bourrage vienne exactement compenser le retrait de matière consécutif à la solidification. Si bourrage et retrait ne sont pas équilibrés (dans un sens ou un autre), des défauts vont apparaître sur les produits: manques ou excès de matière (manques ou bavures), défauts de cotes (problèmes de serrage, quand il s'agit d'une boîte, avec fond et couvercle).

- Les défauts sur les produits consécutifs à un mauvais réglage peuvent être présentés par couples.

2. La course de dosage correspond à la longueur du déplacement de la partie mobile de la machine : plus la distance parcourue est grande, plus la quantité de matière dosée est importante. 
- Le couple striage - brûlure est lié à la phase dynamique d'injection: il y a brûlure quand on injecte trop vite; il y a des stries sur le produit quand on injecte trop lentement.

- Le couple dépressions - cassures est lié à la valeur de la pression de maintien pendant la phase statique: quand la pression de maintien est trop faible, on trouve des dépressions (ou « retassures ») sur les produits; quand elle est trop forte, on a des cassures.

- Le couple manques - bavures provient d'un déséquilibre des pressions entre phase d'injection et phase de maintien: il y a manque quand le bourrage est trop faible, bavure quand il est trop fort.

- Le couple serrage fort - serrage faible provient d'un déséquilibre, dans la phase de maintien, entre bourrage et retrait.

Quand un défaut apparaît sur un produit, l'opérateur peut agir sur un certain nombre de paramètres de réglage. Ces paramètres sont au nombre de 7 :

- Le temps d'injection dynamique (TID): temps pendant lequel s'exerce la pression d'injection

- La pression de commutation (PC) : c'est une norme, habituellement réglée autour de 110 bars, qui fait basculer de la phase dynamique à la phase statique

- La pression de maintien (P2): pression exercée pendant la phase statique, dont la valeur est située entre 20 et $40 \%$ de la pression de commutation

- Le temps de maintien (T2), pendant lequel s'exerce la pression de maintien

- Le temps de refroidissement (T3), temps situé entre maintien et éjection des pièces (le retrait se poursuit, sans être compensé par du bourrage)

- La contre-pression $(\mathrm{CP})$, pression légère (10\% de PC) exercée au moment où s'effectue le dosage de la matière (pour l'homogénéiser).

L'essentiel du travail des régleurs consiste à intervenir en cours de fabrication pour corriger des défauts consécutifs à un réglage qui a bougé. Ces situations sont nombreuses. Mais on peut les classer en deux catégories. Dans les cas les plus simples, la cause du défaut est une variable unique correspondant à un paramètre de réglage. L'exemple typique est celui du couple brûlures - striages : dans ce cas, la cause est le temps d'injection dynamique, TID. C'est donc une relation simple: une cause - un défaut. Mais dans beaucoup d'autres cas, il n'y a pas de relation simple et univoque entre une cause et un défaut. C'est ce qu'on appelle des situations compensées, car plusieurs causes peuvent agir de façon contradictoire sur les défauts. Il peut se trouver des cas où un paramètre déréglé est compensé par un autre paramètre déréglé. Dans ce cas, il n'y a pas de défaut, alors que la machine est en situation de dysfonctionnement. Dans d'autres cas, la compensation n'est pas parfaite, de sorte qu'il y a un défaut (souvent un léger serrage en plus ou en moins), mais un défaut qu'il n'est pas possible de rattacher directement à un seul paramètre. Ces situations, qu'on peut qualifier de systémiques, où deux ou plusieurs paramètres sont la cause d'un ou plusieurs défauts, sont les plus difficiles à traiter. Ces situations de compensation peuvent être regroupées en trois catégories:

- La compensation est due à un déséquilibre entre les paramètres liés à la phase d'injection et ceux liés à la phase de maintien; autrement dit, la compensation vient d'un bourrage déséquilibré au moment de la commutation. Par exemple, une pression de commutation trop faible est compensée par une pression de maintien trop forte.

- La compensation est due à un déséquilibre, dans la phase de maintien, entre bourrage et retrait. Par exemple, une pression de maintien trop faible a pour résultat que le retrait se poursuit pendant le temps de refroidissement, entraînant un serrage insuffisant.

- Il peut y avoir aussi compensation généralisée. En effet, à côté de l'état d'équilibre ou de déséquilibre entre bourrage et retrait, il y a des conditions générales qui viennent aggraver ou annuler ces déséquilibres. C'est notamment le résultat de l'action de la température et de la contre-pression: ce sont des conditions générales aggravantes, atténuantes, voire inhibantes.

Pour gérer ces situations de compensation, les régleurs disposent d'un instrument: la courbe des pressions, qu'ils peuvent faire apparaître sur l'écran de la MOCN. Cette courbe des pressions (cf. 
figure 2) permet d'obtenir la valeur de 5 paramètres de réglage: la pression de commutation PC, le temps d'injection dynamique TID, la pression de maintien P2, le temps de maintien T2, la contrepression $\mathrm{CP}$.

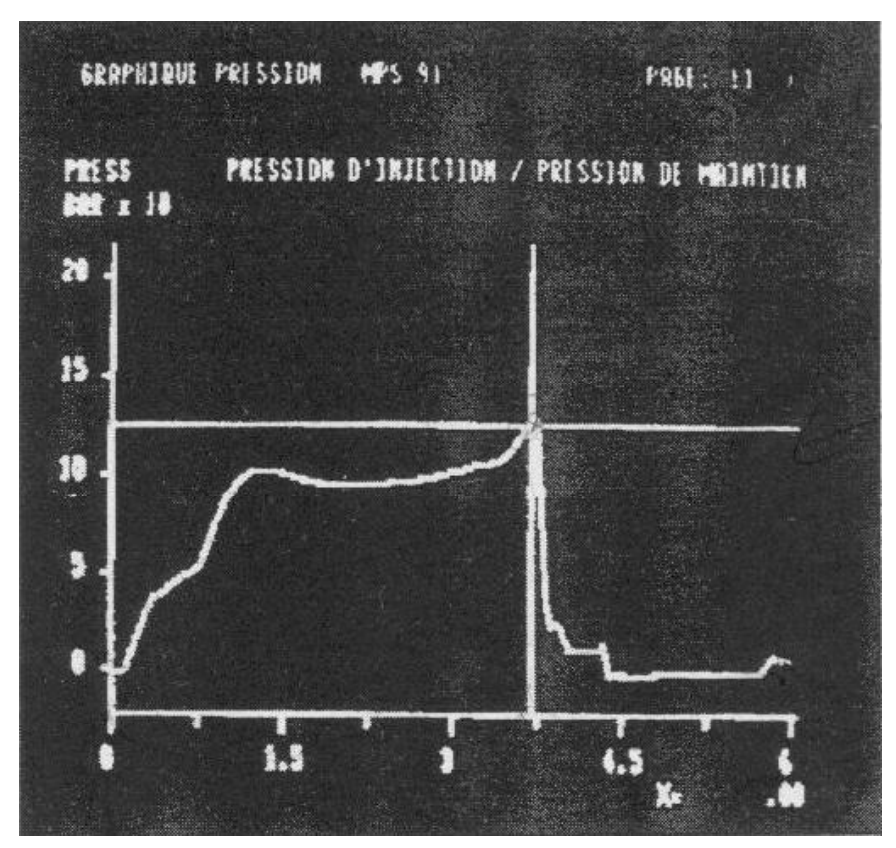

Figure 2: la courbe des pressions

Figure 2: the curve of pressure

\begin{abstract}
Légende: en abscisse, on a des temps (en secondes); en ordonnée, des pressions (en bars). Le point d'intersection des 2 droites désigne la commutation. Son abscisse indique la valeur de TID, son ordonnée la valeur de PC. La contre-pression CP est la variable la plus difficile à lire: elle se manifeste par l'instant où la pression augmente de nouveau très légèrement (sur le graphique à 6 secondes). Le temps de maintien $\mathrm{T} 2$ est donné par la distance entre la commutation et le début de l'action de la contre-pression. La pression de maintien P2 diminue par paliers pendant le temps de maintien.
\end{abstract}

Les régleurs peuvent donc gérer leur action de deux manières : ils peuvent s'appuyer uniquement sur les défauts observés sur les produits et agir sur les paramètres dont ils pensent qu'ils sont la cause du défaut. Nous dirons qu'ils effectuent un pilotage par les défauts. Ils peuvent aussi consulter la courbe des pressions, corriger les paramètres qu'ils estiment déréglés en observant la courbe et contrôler le résultat sur les défauts. Nous dirons qu'ils effectuent un pilotage par la courbe. Évidemment, ils peuvent combiner ces deux sortes de pilotages, en alternant prises d'information sur la courbe et sur les défauts.

\title{
1.3.- Le simulateur
}

Le simulateur a connu plusieurs versions. On présentera la dernière version, élaborée par J. de Frileuze, étudiant en master informatique. Le principe général est le suivant: on se centre exclusivement sur la correction de défauts de réglage apparus en cours de production. L'analyse du travail préalable qui a été effectuée a montré que c'était là l'activité critique des régleurs. On a donc retenu les 7 paramètres de réglage présentés plus haut. On y a ajouté une variable correspondant à un état d'usure d'une pièce de la machine: une buse «matée », par où passe la matière au moment de l'injection. Cette situation entraîne une modification du réglage d'ensemble. L'action à effectuer consiste alors à changer la buse. Avec l'accord de l'expert qui a supervisé l'analyse, on a opéré des simplifications 
dans les paramètres de réglage qui ont été retenus ${ }^{3}$. La principale modification qui a été faite par rapport à la situation professionnelle de référence a consisté, avec l'appui de l'expert, à discrétiser les paramètres d'action qui, dans la réalité, sont des variables continues, mais que les régleurs se représentent sous la forme de classes de situations: par exemple, une pression peut être normale, trop faible ou trop forte. Ceci nous a permis de considérer les problèmes posés comme des problèmes de transformation d'états. On retrouve là une des indications relevées par les théoriciens de la «physique qualitative » (Bobrow, 1984). Ainsi, comme on a retenu 7 paramètres d'action à 3 valeurs, et 1 à 2 valeurs (la buse, matée ou normale), la totalité des valeurs fournies pour les 8 paramètres correspond à 4374 états possibles de la machine (soit 7 puissance 3 pour les 7 paramètres à 3 valeurs x 2, pour le paramètre à 2 valeurs).

Le simulateur fonctionne de la manière suivante: une liste de problèmes est choisie avant le passage des régleurs. Nous avons choisi 17 problèmes, dont le premier avait pour but de familiariser le régleur avec le simulateur. 13 régleurs sont passés sur le simulateur. 5 d'entre eux n'ont traité que la première série des problèmes, soit 9 problèmes. 8 ont traité les deux séries. Tous les problèmes débutent par la présentation des défauts. Le régleur peut soit effectuer un réglage, soit demander la courbe des pressions. Pour un problème donné, le régleur avait pour consigne de s'arrêter quand « il jugeait la situation satisfaisante » (sans plus de précision). Cette consigne, intentionnellement floue, a donné lieu à 3 types de réponses : 1/ Pour les uns, l'arrêt d'un problème implique qu'il n'y ait plus de défauts inacceptables sur les produits, ce qui suppose qu'on acceptera par exemple un léger serrage en plus ou en moins. Les compensations ne sont pas prises en compte. 2/ Pour d'autres, l'arrêt d'un problème coïncide avec l'absence totale de défauts, acceptables ou inacceptables. Les compensations ne sont pas non plus prises en compte. 3/ Enfin, pour la troisième catégorie, l'arrêt du problème implique une absence totale de défauts et de compensations.

\section{4.- Le codage des données}

Le codage du corpus a été fait à partir de 3 principes :

1 - Chaque protocole a été découpé en opérations élémentaires. Chaque opération élémentaire comporte une prise d'information (sur les défauts ou sur la courbe) et une action. L'action est généralement un réglage mais peut être aussi une prise d'information supplémentaire, sur les défauts (notée D) ou sur la courbe (notée C).

2 -Les réglages consistent en des modifications portant sur un des 8 paramètres retenus. La valeur de chaque paramètre, avant et après l'action, est notée de la manière suivante:

- Valeur faible $=-1$

- Valeur normale $=0$

- Valeur forte $=1$

3-On ajoute à ce qui précède les valeurs des paramètres de la machine, qui ne sont pas forcément perçues par le régleur.

On a donc, pour chaque ligne de codage,

- Ce que le régleur voit des défauts,

- Ce que le régleur voit de la courbe, s'il l'a appelée,

- L'état des paramètres de la machine, qui est indépendant des prises d'information du régleur,

- Les actions de réglage sur les paramètres,

- Les opérations de gestion de la tâche.

Tous les protocoles comportent autant de lignes de codage qu'il y a eu d'opérations élémentaires. Voici un exemple de codage d'un protocole correspondant à la résolution d'un problème par un ré-

3. Pour les transformations effectuées, se reporter à Pastré (2004, pp. 24 à 28). 
gleur:

\begin{tabular}{|c|c|c|c|c|c|c|c|c|c|c|c|c|c|c|c|c|c|c|}
\hline \multicolumn{2}{|c|}{$\begin{array}{l}\text { Ce que le sujet peut } \\
\text { voir des défauts, s'il } \\
\text { le demande }\end{array}$} & \multicolumn{3}{|c|}{$\begin{array}{c}\text { Ce que le } \\
\text { sujet peut } \\
\text { voir de la } \\
\text { courbe, s'il le } \\
\text { demande }\end{array}$} & \multicolumn{9}{|c|}{ Etat objectif des variables } & \multicolumn{3}{|c|}{$\begin{array}{l}\text { Action de réglage } \\
\text { (paramètre, sens) }\end{array}$} & \multicolumn{2}{|c|}{$\begin{array}{l}\text { Prise } \\
\text { d'info }\end{array}$} \\
\hline $\begin{array}{l}\text { Défaut } \\
\text { d'aspect }\end{array}$ & $\begin{array}{l}\text { Défaut } \\
\text { de ser- } \\
\text { rage }\end{array}$ & $\mathrm{Ti}$ & $\mathrm{Pc}$ & $\mathrm{Cp}$ & $\mathrm{P} 2$ & $\mathrm{Ti}$ & $\mathrm{Pc}$ & $\mathrm{Cp}$ & $\mathrm{P} 2$ & $\mathrm{~T} 2$ & $\mathrm{~T} 3$ & $\mathrm{~T}^{\circ}$ & $\mathrm{Bu}$ & $\mathrm{R} 1$ & $\begin{array}{l}\text { sens } \\
\text { vala- } \\
\text { ble? }\end{array}$ & $\mathrm{R} 2 \ldots$ & $\mathrm{D}$ & $\mathrm{C}$ \\
\hline Cas* & $\mathrm{S}+1$ & & & & & 0 & 0 & 0 & 1 & 0 & 0 & 0 & 0 & $\mathrm{~T}^{\circ}$ & + & & 1 & \\
\hline B2, cas & $\mathrm{N}$ & & & & & 0 & 0 & 0 & 1 & 0 & 0 & 1 & 0 & $\mathrm{~T}^{\circ}$ & - & & & 1 \\
\hline & & 0 & 0 & 0 & 1 & 0 & 0 & 0 & 1 & 0 & 0 & 0 & 0 & $\mathrm{P} 2$ & - & & 1 & \\
\hline $\mathrm{N}$ & $\mathrm{N}$ & & & & & 0 & 0 & 0 & 0 & 0 & 0 & 0 & 0 & & & & & 1 \\
\hline & & 0 & 0 & 0 & 0 & 0 & 0 & 0 & 0 & 0 & 0 & 0 & 0 & & & & & \\
\hline
\end{tabular}

Tableau 1 : Exemple du codage des données pour un problème

Table 1: Example of coding data for a problem

\section{2.- Les étapes de la recherche}

Cette recherche a commencé entre 1992 et 1994 par un premier travail d'analyse de Pastré, qui a donné lieu à un rapport à destination du Ministère de la Recherche. Il n'était pas prévu de poursuivre ce travail avant que J-F. Richard ne vienne solliciter l'auteur du rapport. Ce premier travail d'analyse

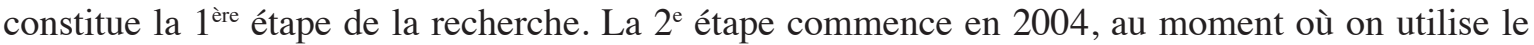
corpus rassemblé plus de 10 ans auparavant pour modéliser les activités de résolution de problèmes selon les deux démarches mentionnées en introduction: la méthode à base des connaissances et la méthode à base des contraintes. La $3^{\mathrm{e}}$ étape apparaît au moment où on compare les résultats obtenus par les modélisations et ce qu'on sait par ailleurs de l'activité réelle des régleurs.

\section{1.- La première étape : pilotage par la courbe, pilotage par les défauts}

L'analyse faite en 1994 a fait apparaître un résultat robuste, qui ne sera plus remis en cause par la suite: il y a deux grands types de stratégies chez les régleurs, qui dépendent de la manière dont ils gèrent la tâche: le pilotage par la courbe et le pilotage par les défauts.

Le pilotage par la courbe est majoritaire: les prises d'information se font à la fois en consultant la courbe des pressions et les résultats sur les produits en termes de défauts. La courbe des pressions est un instrument symbolique qui permet de connaître la valeur de 5 paramètres de réglage: PC, CP, TID, $\mathrm{P} 2$ et T2. Encore faut-il savoir la lire en reconnaissant dans la forme de la figure les 5 variables qui y sont représentées. En combinant les informations présentées sur la courbe et les informations fournies par l'observation des défauts, les régleurs sont à même de résoudre tous les problèmes présentés, y compris ceux qui comportent des compensations. Le pilotage par la courbe est le fait des régleurs les plus compétents. Cela n'exclut pas des erreurs ponctuelles dans le processus de résolution, mais ces erreurs sont finalement corrigées. On aboutit ainsi à des résultats où il n'y a pas de défauts sur les produits et où la machine fonctionne de façon satisfaisante (pas de compensation). Remarquons toutefois qu'aucun des régleurs observés n'a réussi à éliminer toutes les compensations: plus la difficulté du problème augmente, plus la probabilité de laisser des compensations augmente également.

4-Le pilotage par les défauts est une stratégie minoritaire chez les régleurs. Les prises

d'information se font cette fois uniquement par consultation des défauts produits. Cela ne veut pas dire que ces régleurs fonctionnent simplement par essais et erreurs. Le régleur qui est le plus 
représentatif de cette stratégie maîtrise bien les connaissances du domaine: il sait par exemple qu'une bavure légère a toutes les chances d'être due à un excès de température ou qu'une brûlure est due à une vitesse d'injection trop élevée. Il arrive donc à corriger les défauts présents dans chacun des problèmes, même s'il a tendance à laisser passer les défauts jugés acceptables (notamment pour le serrage). Le problème de cette stratégie est celui des compensations, surtout des compensations parfaites: quand deux paramètres compensent leurs effets, le pilotage par les défauts ne fournit aucun moyen pour repérer les situations compensées.

\section{2.- Deuxième étape: modélisation du régleur idéal}

Le projet «Plastur » S'inscrit dans ce contexte. À la suite de la première analyse, il a semblé naturel de construire un modèle de « régleur idéal », pouvant servir de référence pour analyser les stratégies des régleurs réels. Cette nouvelle étape se situe dans la continuité de la précédente, en reprenant notamment à son compte la distinction entre les stratégies de pilotage par la courbe et la stratégie de pilotage par les défauts. Notons par ailleurs qu'elle a été réalisée par les chercheurs qui travaillaient sur une modélisation à base de connaissances ${ }^{4}$. Comment se représente-t-on le fonctionnement d'un « régleur idéal »? Si on veut à coup sûr éliminer tous les défauts (de réglage, ne l'oublions pas) sur le simulateur, il suffit de corriger tous les paramètres déréglés. Rappelons que nous en avons retenu 8 La valeur de 5 de ces paramètres est donnée par lecture de la courbe des pressions. On peut donc corriger à coup sûr PC, CP, TID, P2 et T2. À ce moment de la résolution du problème, de deux choses l'une: ou bien il ne reste plus de défauts sur les produits et on est pratiquement sûr, à une exception près ${ }^{5}$, de ne plus avoir de compensation. Ou bien, une fois la courbe bien réglée, il reste un défaut et on peut, par inférence, chercher la cause du côté des 3 paramètres non représentés sur la courbe: T3, le temps de refroidissement, $\mathrm{T}^{\circ}$, la température de la matière, et la buse matée. Mais alors on n'a plus affaire à 4374 états de la machine, mais à 18 ( $\left.3^{2} \times 2\right)$. Parmi les possibles qui restent à sa disposition, le régleur idéal choisit alors l'opération qui a la fréquence la plus élevée.

Si maintenant nous comparons les stratégies des régleurs réels à ce modèle du régleur idéal, nous retrouvons les deux stratégies du pilotage par la courbe et du pilotage par les défauts. On a d'un côté une stratégie caractérisée par le fait que ces régleurs n'appellent jamais la courbe, ne pilotent que par les défauts et laissent passer les compensations. Trois régleurs se rapprochent le plus de ce modèle (Lucien, Henri et Jean ${ }^{6}$ ): pour ces trois sujets la qualité de la simulation de la stratégie est très bonne. D'un autre côté, on trouve une stratégie de pilotage par la courbe et de contrôle par les défauts. Elle ne laisse passer que très peu de compensations. Trois opérateurs (Jacques, Charles, François) ont un comportement proche de ce régleur idéal; les autres utilisent la démarche, en appelant notamment la courbe de façon fréquente, mais ils continuent à commettre des erreurs en cours de problème et laissent passer des compensations. C'est pourquoi la qualité de la simulation pour ce type de stratégie n'est pas complètement satisfaisante: elle ne permet que de rendre compte d'environ $60 \%$ des problèmes traités avec cette stratégie.

\section{3.- Troisième étape : l'apport des modélisations}

Pour avoir une idée précise des deux formes de modélisation utilisées pour cette recherche, nous invitons le lecteur à se reporter à l'article de Labat et al. (2007), pour la modélisation à base de connaissances, et à Richard et al. (à paraître), pour la modélisation à base de contraintes. Nous nous contenterons ici de présenter ce qu'apporte la démarche de modélisation par les contraintes par rapport à l'analyse des stratégies des acteurs.

1-Il existe non pas deux, mais trois grands types de stratégies, selon la manière de gérer la tâche:

4. Pour plus de détails concernant cette modélisation, voir Labat et al. (2007).

5. La seule exception est une compensation qui s'établirait entre une buse matée et une température élevée.

6. Les 13 régleurs étudiés ont été désignés par une lettre $(\mathrm{A}, \mathrm{B}, \mathrm{C} \ldots)$, pour respecte l'anonymat. Pour rendre le texte moins abstrait, on a attribué un prénom à chaque lettre: de $\mathrm{A}$, André à $\mathrm{M}$, Michel. 
la conduite par les défauts (D), la conduite par la courbe (C), la conduite par la courbe et les défauts $(\mathrm{CD})$.

2-Les connaissances du domaine sont variables chez les régleurs, même quand ils mobilisent la même stratégie. On peut penser que la stratégie effective de chaque régleur dépend de la combinaison de 2 variables: la gestion de la tâche et le niveau de connaissances du domaine.

3 -On peut utiliser la courbe dans sa stratégie sans avoir une connaissance complète de lecture de la courbe. Il existe donc deux domaines de connaissances: des connaissances portant sur les causes des défauts et des connaissances portant sur la lecture de la courbe.

\section{3.- Retour sur les stratégies des régleurs}

\section{Passer des règles aux stratégies}

On trouvera en annexe la liste des règles qui permettent de modéliser les protocoles de résolution des problèmes des régleurs selon l'approche par les contraintes. Ces règles se regroupent en trois blocs: $1 /$ les règles qui relèvent de la gestion de la tâche (prendre de l'information sur les défauts ou sur la courbe ou effectuer un réglage), 2/ les règles qui indiquent l'action de réglage à effectuer en fonction des connaissances du sujet et de l'information qu'il a prise, $3 /$ les règles recourant à des heuristiques générales quand l'opération effectuée est jugée non satisfaisante par le régleur. On trouvera également en annexe un exemple de résolution d'un problème avec explicitation des règles utilisées. La modélisation de l'activité par les contraintes a permis d'énumérer les différentes règles utilisées par les régleurs. Mais nous pouvons faire un pas de plus dans une perspective de didactique professionnelle: faire l'hypothèse que les règles utilisées par un régleur, quel que soit le problème, peuvent être regroupées pour former une configuration cohérente. Nous appellerons «stratégie » une certaine organisation de l'activité propre à un régleur qu'on peut retrouver dans toutes ses résolutions de problèmes. Cette stratégie peut être exprimée par un ensemble restreint de « méta-règles » qui permettent de rendre compte de l'ensemble des règles sélectionnées par le régleur. Cet ensemble de méta-règles porte sur 3 domaines: 1/ la gestion de la tâche, 2/ le niveau de maîtrise des connaissances du domaine, 3/ l'importance du recours à des heuristiques générales en cas d'échec en cours de problème. La gestion de la tâche constitue l'élément central qui caractérise une stratégie : pilotage par les défauts $\mathrm{D}$, pilotage par la courbe $\mathrm{C}$, pilotage par la courbe et les défauts $\mathrm{CD}$. Mais à chaque stratégie doit être associé un niveau de connaissances, pour que la stratégie soit efficace : pour une stratégie D, il faut connaître les règles de base du métier qui permettent d'envisager toutes les causes possibles pour un défaut donné. Pour une stratégie $\mathrm{C}$ ou $\mathrm{CD}$, il faut en plus avoir les connaissances nécessaires pour savoir lire la courbe. Chaque fois qu'une stratégie mobilisée n'est pas accompagnée de toutes les connaissances qui la rendent efficace, les régleurs vont faire appel à des heuristiques pour compenser ce manque. Bien entendu, une stratégie peut évoluer en cours d'exercice, notamment chez les régleurs qui n'ont pas encore stabilisé leur niveau de compétence.

Nous présenterons successivement parmi les stratégies observées 1/ celles qui sont stabilisées, 2/ celles qui sont en évolution.

\section{1.- Stratégies stabilisées caractéristiques : Charles, Lucien, Jacques}

Le tableau 1 présente d'abord les 3 cas de régleurs les plus représentatifs des stratégies C, CD et $\mathrm{D}$. Nous indiquons, pour chaque stratégie, la méta-règle de gestion de la tâche, le niveau de connaissances du domaine possédé par le régleur concernant la courbe et les paramètres hors courbe, la règle de fin de traitement utilisée. Nous n'avons pas précisé le recours aux heuristiques générales, parce qu'il est inversement proportionnel au niveau de connaissances du domaine: plus le niveau de connaissances sur l'effet des paramètres est faible, plus les 
heuristiques sont nombreuses.

\begin{tabular}{|c|c|c|c|c|}
\hline Régleur & Gestion de la tâche & $\begin{array}{c}\text { Connaissance de la } \\
\text { courbe }\end{array}$ & $\begin{array}{l}\text { Connaissance des } \\
\text { paramètres hors } \\
\text { courbe }\end{array}$ & Règle d'arrêt du traitement \\
\hline Charles & $\begin{array}{l}\text { C: Appeler la courbe et la } \\
\text { corriger jusqu'au bout. Si plus } \\
\text { de défauts, problème résolu. } \\
\text { Si défauts, agir sur } 1 \text { des } 3 \\
\text { paramètres hors courbe }\end{array}$ & $\begin{array}{l}\text { P2, T2, CP maîtrisés } \\
\text { TID partiellement } \\
\text { PC non maîtrisée }\end{array}$ & $\begin{array}{l}\mathrm{T} 3, \mathrm{~T}^{\circ}, \text { Buse: erreurs } \\
\text { majoritaires (recours à } \\
\text { heuristiques) }\end{array}$ & $\begin{array}{l}\text { Règle } 1: \text { ' 'arrêter en } \\
\text { l'absence de défauts, } \\
\text { même acceptables, et de } \\
\text { compensations } \\
\text { Echec selon } \mathrm{R} 1=1 / 17 \\
\text { Echec selon } \mathrm{R} 2=0 \\
\end{array}$ \\
\hline Lucien & \begin{tabular}{|l} 
D: Prendre uniquement \\
l'information sur les défauts (ne \\
jamais appeler la courbe), agir \\
sur la cause du défaut la plus \\
probable
\end{tabular} & Hors sujet & $\begin{array}{l}\text { Maîtrise de P2, T2, } \\
\mathrm{T}^{\circ} ; \mathrm{T}^{\circ}, \mathrm{TID} \text {, buse }\end{array}$ & $\begin{array}{l}\text { Règle } 3: \text { S'arrêter en } \\
\text { l'absence de défauts } \\
\text { inacceptables (laisser les } \\
\text { défauts acceptables et les } \\
\text { compensations) } \\
\text { Echec selon R1 }=7 / 8 \\
\text { Echec selon R2 = } 5 \\
\text { Echec selon R3 = } 1\end{array}$ \\
\hline Jacques & \begin{tabular}{|l|} 
CD, variante intégrée: \\
Appeler la courbe, la corriger \\
et contrôler le résultat sur les \\
défauts. Si situation améliorée, \\
continuer à corriger la courbe. \\
Si situation dégradée, revenir \\
en arrière en repositionnant le \\
paramètre modifié. Si courbe \\
correcte et persistance de \\
défauts, modifier 1 ou plusieurs \\
paramètres hors courbe
\end{tabular} & $\begin{array}{l}\text { P2, T2, CP maîtrisés } \\
\text { TID et PC maîtrisés } \\
\text { avec quelques erreurs }\end{array}$ & $\begin{array}{l}\mathrm{T} 3, \mathrm{~T}^{\circ} \text {, buse : maîtrise } \\
\text { avec quelques erreurs }\end{array}$ & $\begin{array}{l}\text { Règle } 1 \\
\text { Echec selon } \mathrm{R} 1=1 / 17 \\
\text { Echec selon } \mathrm{R} 2=0\end{array}$ \\
\hline François & $\begin{array}{l}\text { CD, variante éclatée: Appeler la } \\
\text { courbe, la corriger et contrôler } \\
\text { le résultat sur les défauts. Si } \\
\text { situation dégradée, passer à un } \\
\text { pilotage défaut en agissant sur } \\
\text { le paramètre le plus probable }\end{array}$ & $\begin{array}{l}\text { P2, T2, TID maîtrisés } \\
\text { CP maîtrisée partiel- } \\
\text { lement } \\
\text { PC non maîtrisée }\end{array}$ & $\begin{array}{l}\text { Buse: maîtrise } \\
\text { partielle } \\
\mathrm{T}^{\circ}, \mathrm{T} 3 \text { : non maîtrisés }\end{array}$ & $\begin{array}{l}\text { Règle } 1 \\
\text { Echecs selon } \mathrm{R} 1=3 / 17 \\
\text { Echecs selon } \mathrm{R} 2=2 \\
\text { Echec selon R3 = 1 }\end{array}$ \\
\hline André & $\begin{array}{l}\text { CD (variante DC): Commencer } \\
\text { par pilotage défauts, revenir en } \\
\text { arrière si aggravation défaut ou } \\
\text { pas d'effet, appeler la courbe en } \\
\text { cas d'impasse }\end{array}$ & $\begin{array}{l}\text { Connaissances } \\
\text { instables sur P2 et PC }\end{array}$ & $\begin{array}{l}\text { Aucune connaissance } \\
\text { totalement maîtrisée } \\
\text { (même P2, T2, T3). } \\
\text { Recours massif à des } \\
\text { heuristiques générales }\end{array}$ & $\begin{array}{l}\text { Règle } 1 \\
\text { Echecs selon R1 = 7/9 } \\
\text { Echecs selon R2 et R3 =0 }\end{array}$ \\
\hline $\begin{array}{l}\text { Christophe } 1 \\
\text { (pbs } 1 \text { à 10) }\end{array}$ & $\begin{array}{l}\text { CD, variante éclatée: Appeler la } \\
\text { courbe, la corriger et contrôler } \\
\text { le résultat sur les défauts. Si } \\
\text { situation dégradée, passer à un } \\
\text { pilotage défaut en agissant sur } \\
\text { le paramètre le plus probable }\end{array}$ & $\begin{array}{l}\text { CP et TID maîtrisés } \\
\text { avec quelques erreurs } \\
\text { P2, T2, PC non mâ̂- } \\
\text { trisés }\end{array}$ & $\begin{array}{l}\mathrm{T}^{\circ}, \mathrm{T} 3 \text {, buse : erreurs } \\
\text { majoritaires }\end{array}$ & $\begin{array}{l}\text { Règle } 1 \\
\text { Echec selon } \mathrm{R} 1=1 / 10\end{array}$ \\
\hline $\begin{array}{l}\text { Christophe } 2 \\
\text { (pbs } 11 \text { à } 17)\end{array}$ & $\begin{array}{l}\text { CD, variante intégrée } \\
\text { (cf. Jacques) }\end{array}$ & $\begin{array}{l}\text { P2, T2 maîtrisés } \\
\text { TID, CP, PC maîtrisés }\end{array}$ & Paramètres maîtrisés & $\begin{array}{l}\text { Règle } 1 \\
\text { Echec selon } \mathrm{R} 1=0\end{array}$ \\
\hline $\begin{array}{l}\text { Daniel } 1 \\
\text { (pbs } 1 \text { à 9) }\end{array}$ & $\begin{array}{l}\text { CD (variante DC): Prendre } \\
\text { connaissance des défauts et } \\
\text { appeler la courbe, mais utiliser } \\
\text { les défauts pour piloter. Arrêt } \\
\text { quand on obtient une absence de } \\
\text { défauts ou un défaut acceptable }\end{array}$ & $\begin{array}{l}\text { Aucun des paramètres } \\
\text { n'est maîtrisé }\end{array}$ & $\begin{array}{l}\text { Aucun paramètre } \\
\text { maîtrisé }\end{array}$ & $\begin{array}{l}\text { Règle } 3 \\
\text { Echec selon R1 = 8/9 } \\
\text { Echec selon R2 = 3 } \\
\text { Echec selon R3 = 0 }\end{array}$ \\
\hline $\begin{array}{l}\text { Daniel } 2 \\
\text { (pbs } 10 \text { à } 14)\end{array}$ & $\begin{array}{l}\text { CD, variante éclatée } \\
\text { (cf. François) }\end{array}$ & $\begin{array}{l}\text { Maîtrise de P2, T2, } \\
\text { TID } \\
\text { Quelques erreurs sur } \\
\text { CP et PC }\end{array}$ & $\begin{array}{l}\text { Paramètres non } \\
\text { maîtrisés }\end{array}$ & $\begin{array}{l}\text { Règle } 1 \\
\text { Echec selon } \mathrm{R} 1=2 / 5\end{array}$ \\
\hline $\begin{array}{l}\text { Daniel } 3 \\
\text { (pbs } 15 \text { à 17) }\end{array}$ & $\begin{array}{l}\text { CD, variante intégrée } \\
\text { (cf. Jacques) }\end{array}$ & Idem & Paramètres maîtrisés & $\begin{array}{l}\text { Règle } 1 \\
\text { Echecs selon R1 = } 0\end{array}$ \\
\hline
\end{tabular}

Tableau 2: Les trois stratégies utilisées par les régleurs

\section{Table 2: The three strategies used by regulators}

Les régleurs peuvent avoir une stratégie stable pour tous les problèmes: par 
exemple Charles (stratégie C), Lucien (stratégie D), Jacques (stratégie CD intégrée). D'autres régleurs évoluent dans leur stratégie: par exemple Daniel. On a noté chaque étape par un numéro (Daniel 1, Daniel 2, etc.).

Il faut remarquer que sur les régleurs étudiés, on observe un seul cas pour les stratégies $\mathrm{C}$ et $\mathrm{D}$ (Charles et Lucien). Dans ces deux cas, le niveau de connaissances du domaine est bon, avec une différence entre les deux : pour Lucien (stratégie D), il n'y a pas lieu d'indiquer ses connaissances portant sur la courbe, puisqu'il ne l'utilise pas. Pour Charles (stratégie C), on peut distinguer les connaissances qu'il a de la courbe et celles des effets des paramètres hors courbe. Tous les autres régleurs (11 sur 13) relèvent de la stratégie $C D$, mais avec des différences importantes. Cette stratégie $C D$ est la plus complexe. On y voit notamment apparaître une règle que nous appellerons la règle de la situation dégradée: la situation est jugée dégradée lorsque, après une action, apparaissent des défauts plus graves que ceux qui existaient auparavant. Par exemple, on avait un serrage légèrement trop faible (S-1), on obtient un manque ou une bavure, ou un serrage trop faible inacceptable (S-2). Dans ce cas, le régleur revient en arrière (règle d'heuristique générale : si le défaut s'accroît, rétablir le paramètre modifié dans son état antérieur): il prend à nouveau de l'information sur la courbe et les défauts et évalue la cohérence entre les deux. Mais il faut ajouter un point important: si la grande majorité des régleurs relèvent de la stratégie $\mathrm{CD}$, il y a entre eux des différences notables. D'une part, le niveau des connaissances du domaine diffère d'un régleur à l'autre. D'autre part, une analyse plus fine montre qu'il y a plusieurs modalités de stratégie CD et qu'il vaut la peine de les observer dans le détail.

\section{2.- Les trois formes de stratégies CD : Jacques, François, André}

a- La stratégie CD intégrée: elle est représentée de façon prototypique par Jacques: le pilotage se fait principalement par la courbe, mais quand apparaît une situation perçue comme dégradée (accentuation du défaut qu'on essaie de corriger ou apparition de défauts plus graves), le régleur recourt à un contrôle par les défauts, qui fonctionne comme un test d'hypothèse: il s'agit de savoir si on a réussi à transformer le problème en un problème simple (1 défaut -1 cause), pour lequel un pilotage par les défauts est pertinent; ou si on a toujours affaire à un problème complexe (multi-causes, multi-effets, avec des compensations impliquant des paramètres hors courbe), pour lequel le retour vers la courbe est nécessaire, tout en n'étant pas suffisant. Ajoutons, pour compléter cette présentation, que le premier problème traité par Jacques ${ }^{7}$ est intéressant à analyser dans le détail. C'est en effet le seul cas où Jacques commence par modifier directement un paramètre sans consulter la courbe et en contrôlant le résultat sur les défauts. On aurait donc ici un début de stratégie $\mathrm{D}$, où le régleur perçoit une relation saillante entre une cause probable et un défaut (Cas, $\mathrm{S}+1 \Rightarrow$ baisser T3). Mais au vu du résultat (T3 n'est pas ici la cause des défauts), Jacques change de stratégie, appelle la courbe et modifie P2. Il n'est pas impossible que ce choix de stratégie soit assez représentatif des pratiques professionnelles des bons régleurs dans l'atelier. On pourrait le formuler ainsi: si on pense avoir affaire à un problème simple ( 1 cause probable -> un défaut), modifier le paramètre qu'on estime être la cause. Si ça marche, le problème est résolu. Si ça ne marche pas, alors c'est qu'on est en face d'un problème complexe, systémique; il faut alors passer par la consultation de la courbe. Ceci nous amène à formuler une hypothèse qui permet d'expliquer un certain nombre de détails de traitement chez plusieurs régleurs. Il y aurait deux grands types de stratégies: une stratégie dominante dans l'atelier, quand les régleurs sont aux prises avec des problèmes simples; et une stratégie dominante dans la simulation, quand les régleurs doivent traiter des problèmes systémiques. En effet, en simulation nous avons choisi de multiplier les problèmes complexes, systémiques, avec quelquefois des compensations impliquant 4 variables. Par contre, dans le travail réel, ce sont les problèmes simples, où un seul paramètre est déréglé et constitue la cause du défaut, qui sont de loin les plus fréquents ${ }^{8}$. On comprend alors la logique de Jacques: pour le premier problème qu'il a traité, il a

7. On rappelle que Jacques est considéré comme le meilleur régleur de l'entreprise.

8. Dans les ateliers on parle souvent de la « loi des 80/20 », appelée « loi de Pareto »: $80 \%$ des problèmes rencontrés sont des problèmes simples, mobilisant $20 \%$ de temps et d'activité cognitive. $20 \%$ des problèmes sont des problèmes complexes, mobilisant $80 \%$ des ressources. Or, sur le simulateur, on est plus proche d'un rapport inversé: $80 \%$ 
utilisé naturellement la stratégie de l'atelier: faire une première opération en supposant un problème simple; la traiter comme un test d'hypothèse et, d'après le résultat, choisir la stratégie "problème simple », c'est-à-dire la stratégie D, ou la stratégie " problème complexe », c'est-à-dire la stratégie majoritairement C. Mais il a vite compris que sur simulateur il était plus intelligent de faire l'hypothèse de problèmes complexes majoritaires, donc de commencer par appeler la courbe, pour faire un diagnostic de situation. Ce qui corrobore cette hypothèse est le fait qu'à mesure du déroulement des problèmes Jacques se rapproche de plus en plus du type de stratégie C (corriger d'abord la courbe, puis les défauts s'il en reste), avec cette seule différence avec Charles qu'il appelle plus souvent un contrôle par les défauts. Cette question du rapport entre situation d'atelier et situation de simulation sera discutée plus loin.

b- La stratégie CD éclatée est représentée par François (cf. tableau 2). Elle est caractérisée par la règle de la situation dégradée. Le régleur pilote par la courbe, mais contrôle en permanence par les défauts. Il évalue on line le résultat de chaque opération en cherchant à savoir s'il avance dans la résolution de son problème ou non. Il combine deux démarches, l'une qui s'appuie sur une représentation symbolique externe de la situation (la courbe des pressions), l'autre qui regarde directement le résultat de chaque opération sur l'évolution des défauts. Et quand il perçoit une contradiction entre ces deux démarches, c'est-à-dire quand il perçoit une dégradation de la situation, il donne priorité au pilotage par les défauts et en revient à une démarche de poursuite: le but étant "zéro défauts », toute opération qui éloigne du but est à écarter. Dans le premier type de démarche, le régleur s'appuie sur une représentation symbolique abstraite de la situation pour conduire son action. Dans le deuxième type de démarche il évalue en permanence l'évolution de l'écart entre le but recherché et le résultat de ses opérations. Remarquons que ce qui caractérise la stratégie CD dans son ensemble est la combinaison des deux démarches, symbolique et empirique. Dans le cas des stratégies CD éclatées, en cas de perception d'une contradiction, c'est finalement la démarche empirique qui l'emporte. Remarquons enfin que cette stratégie CD éclatée est celle qui est observée le plus fréquemment dans notre population de régleurs.

c- La pseudo-stratégie CD (ou stratégie DC) est représentée par André (cf. tableau 2). Elle est en quelque sorte intermédiaire entre la stratégie D et la stratégie CD éclatée. En fait elle consiste en un pilotage par les défauts, avec des incursions, plus ou moins heureuses, du côté de la courbe. On a choisi de la représenter par André, dont la caractéristique est de posséder des connaissances très incertaines du domaine. Plusieurs régleurs procèdent selon cette pseudo-stratégie CD. Leur point commun est une absence de connaissances solides dans la lecture de la courbe. D'où un traitement, parfois heureux, le plus souvent malheureux, des informations fournies par la courbe. Par contre, ils se différencient par la maîtrise plus ou moins grande des connaissances portant sur les causes possibles des défauts. André par exemple maîtrise mal ces connaissances. D'autres le font mieux que lui: ils se rapprochent de ce fait de la stratégie $\mathrm{D}$ de Lucien, qui, rappelons-le, possède une très bonne connaissance des causes possibles des défauts.

\section{3.- les stratégies en évolution}

Jusqu'à maintenant nous avons fait comme si chaque régleur mobilisait la même stratégie du début à la fin de l'exercice. C'est vrai pour Charles et Lucien. Il faut d'ailleurs remarquer qu'ils sont les seuls, chacun dans son genre, à mobiliser l'un la stratégie C, l'autre la stratégie D. C'est également le cas de Jacques, qui, étant donné le type des problèmes posés, n'avait plus beaucoup de progression possible (effet de seuil). La plupart des autres régleurs ont évolué dans leur stratégie en cours d'exercice. Ils l'ont fait plus ou moins fortement, notamment ceux qui ont pu faire les deux séries d'exercices (17 problèmes traités au total). La palme de la progression revient à deux jeunes régleurs, Christophe et Daniel, qui, en plus, ont bénéficié de la séance de formation sur simulateur qui a eu lieu entre les deux passations. Christophe débute avec une stratégie CD éclatée (cf. Christophe 1, tableau 2) et termine avec une stratégie CD intégrée (cf. Christophe 2, tableau 2), qui le met au même

de problèmes complexes, $20 \%$ de problèmes simples. 
niveau de performances que Jacques. Daniel fait encore mieux : ce régleur débutant, doté d'un BTS de plasturgie, commence par une pseudo-stratégie CD qui le positionne au même niveau de performance qu'André. Il bascule ensuite vers une stratégie CD éclatée et termine avec une stratégie $C D$ intégrée (cf. Daniel 1 et 3, tableau 2). Les autres régleurs ont des progressions moins spectaculaires. Mais chez tous ceux qui progressent on voit apparaître une constante: la part d'empirique a tendance à diminuer, sans jamais disparaître; la part de symbolique a tendance à augmenter. Et il y a un ordre entre les 3 stratégies $C D$ : on pratique d'abord la pseudo $C D$, ensuite $C D$ éclatée, enfin $C D$ intégrée.

\section{4.- Discussion}

Commençons par rappeler les principaux résultats de cette recherche en termes d'identification des stratégies des acteurs:

1 - Les stratégies $\mathrm{D}$ et $\mathrm{C}$ représentent des cas uniques : on peut les considérer comme assez exceptionnelles. Toutes les deux sont mobilisées par un seul régleur. Ceux-ci utilisent ces stratégies de façon stable, avec pour chacun un bon niveau de connaissances, ce qui conforte la stabilité du modèle opératif de ces deux régleurs.

2-Chez tous les autres régleurs, la stratégie mobilisée, $\mathrm{CD}$, consiste à combiner deux démarches dans des proportions diverses: on pilote en s'appuyant sur les données factuelles fournies par le résultat des actions, qui permettent de savoir si on se rapproche ou si on s'éloigne du but (ou si on n'a pas bougé par rapport au but): c'est une démarche empirique. Mais on pilote également en s'appuyant sur une représentation symbolique externe, qui fournit, pour qui sait la lire, la valeur de 5 paramètres sur 8 et qui seule permet de traiter efficacement les situations compensées: c'est une démarche symbolique.

3 - La stratégie $\mathrm{CD}$ prend 3 formes principales: une stratégie pseudo- $\mathrm{CD}$, une stratégie CD éclatée, une stratégie $\mathrm{CD}$ intégrée. Chacune de ces formes représente un niveau de compétence dans l'activité de réglage: les régleurs qui fonctionnent en pseudo-CD ont un niveau de compétence et de performance faible; ceux qui fonctionnent en CD éclatée ont un niveau de compétence honnête mais pourraient mieux faire; ceux qui fonctionnent en $C D$ intégrée sont les régleurs les plus compétents.

4-Quand on observe les régleurs qui ont évolué dans leur stratégie, on constate que l'évolution se fait toujours dans le même ordre : la part d'empirique a tendance à diminuer; la part de symbolique a tendance à augmenter, au point qu'in fine la stratégie CD intégrée en vient à se confondre avec la stratégie $\mathrm{C}$ représentant le «régleur idéal ».

Passons maintenant à un essai d'interprétation de ces données.

\section{1.- Stratégie d'atelier et stratégie de simulation}

Il est intéressant de revenir sur la distinction introduite entre stratégies d'atelier et stratégies de simulation. Sur un simulateur, on a tendance à mettre en scène des situations critiques, complexes, atypiques, voire des situations qu'on ne risque pas trop de rencontrer dans le réel, mais qui font néanmoins partie du champ des possibles. Pensons à l'usage des simulateurs dans la formation des conducteurs professionnels de centrales nucléaires: certaines des situations utilisées (par exemple une fuite radioactive avec perte d'une partie des instruments de conduite, cf. Pastré, 2005) n'ont quasiment aucune « chance » de se produire dans le réel. Il faut bien pourtant que les opérateurs apprennent à les maîtriser. D'où un biais lié à la simulation: on risque de ne retenir que les situations atypiques. C'est ce qui s'est produit avec le simulateur de réglage de presses à injecter: nous avons inversé la proportion entre problèmes simples (un défaut, une cause) et problèmes complexes (multi-causes, multi-effets, avec compensations). Les régleurs n'ont pas été dupes. Ils ont adapté leur stratégie à la situation de simulation en choisissant d'emblée, quand ils étaient capables de le faire, une stratégie $\mathrm{CD}$ intégrée, comme on le voit dans le cas de Jacques: quand il aborde le premier problème, il utilise 
spontanément une stratégie $\mathrm{D}$, en pensant a priori qu'il est en face d'une problème simple, qui est beaucoup plus probable ( «loi des 80/20») qu'un problème systémique. Mais il comprend vite que, dans la simulation, on a donné la priorité aux problèmes systémiques. Il adapte donc sa démarche en conséquence: pour les problèmes suivants, il fait l'hypothèse qu'il est face à des problèmes systémiques, et choisit la stratégie (CD intégrée) qui leur est adaptée.

On pourrait alors se demander si la transposition effectuée par la simulation entre la situation professionnelle de référence et la situation didactique n'a pas transformé la nature des problèmes posés: il ne s'agirait plus de problèmes écologiques, mais de problèmes académiques. Un argument qui va dans ce sens consiste à prendre en compte le but que se donnent les acteurs. Nous avons vu que, étant donné la consigne intentionnellement ambiguë qui était formulée («trouver une solution satisfaisante »), une grande latitude était laissée à l'interprétation des régleurs. Ainsi nous avons trouvé trois interprétations: 1 / certains considèrent le problème résolu quand il ne reste plus de défauts inacceptables, alors même qu'il peut subsister des défauts acceptables (par exemple, un léger serrage) ou des compensations. 2/ D'autres considèrent le problème résolu quand il n'y a plus de défauts, même acceptables, mais sans tenir compte de la persistance éventuelle d'une compensation. 3/ D'autres enfin considèrent le problème résolu quand il ne reste plus ni défauts ni compensations. Ainsi la catégorie 1 correspondrait à un objectif pragmatique, reproduisant les pratiques d'atelier: on arrête de chercher quand on a un produit acceptable. Quant à la catégorie 3, elle correspondrait à la transformation d'un problème pratique de fabrication en un problème académique de résolution de problème. Mais ce serait oublier que, dans la réalité du travail, il existe aussi bien des situations qui sont solubles par des stratégies simples que des situations qui impliquent nécessairement le recours à des stratégies de type $\mathrm{CD}$ intégrée. En effet, dans le travail réel, les cas sont fréquents où on a à la fois un défaut inacceptable et une situation de compensation. Dans ce cas, on ne peut pas se contenter de s'arrêter aux défauts, acceptables ou non; la seule solution acceptable correspond dans ce cas à une suppression des compensations et des défauts. C'est pourquoi la vraie différence entre situations « écologiques » et situations « académiques » ne porte pas sur la nature des problèmes, mais uniquement sur la proportion entre problèmes simples et problèmes systémiques : $80 \%$ de problèmes simples dans l'atelier, $80 \%$ de problèmes systémiques sur simulateur. C'est la raison pour laquelle Jacques montre bien qu'il maîtrise les deux types de stratégies: D pour les problèmes simples, CD intégrée pour les problèmes systémiques. La seule « erreur » qu'il lui arrive de commettre est dans le choix, a priori, de la stratégie mobilisée. Et cette erreur est corrigée tout de suite dès qu'il découvre le résultat de sa première opération. Mais l'important est que son « modèle opératif » (Pastré, 2004), c'est-à-dire la manière dont il organise son activité grâce à la conceptualisation qu'il fait de la situation, comporte ces deux stratégies, qu'il a à disposition, en tant que de besoin.

\section{2.- Les deux sources des stratégies mobilisées}

Comparons maintenant le modèle opératif de Jacques, tel qu'il ressort de notre analyse, à celui de François. Celui-ci est capable de mobiliser une stratégie CD éclatée: il recourt à la fois aux défauts et à la courbe, mais quand il y a contradiction entre les deux, il finit toujours par privilégier les défauts. Ajoutons à cela que François ne dispose pas de tout le répertoire de connaissances, ni sur le domaine, ni sur la courbe, susceptibles de rendre efficiente sa stratégie. Autrement dit, alors que Jacques a non seulement à sa disposition deux stratégies adaptées aux problèmes, mais également les moyens de les mettre en œuvre, ce n'est pas le cas de François, qui n'a pas tous les moyens conceptuels nécessaires pour mener jusqu'au bout une stratégie CD intégrée. C'est pourquoi, confronté à une contradiction, il revient en arrière vers une stratégie de type $\mathrm{D}$. Cela veut dire que, dans le choix ponctuel d'une stratégie, deux facteurs sont en cause: la décision de choisir l'une ou l'autre, mais aussi la capacité de les mettre en œuvre.

Ceci nous amène à apporter une précision. On aurait tendance à assimiler pilotage par la courbe et stratégie CD intégrée, comme si le niveau de conceptualisation requis par la stratégie CD intégrée équivalait à l'utilisation d'un instrument symbolique: la courbe des pressions. Or dans une recherche 
antérieure (Pastré, 1992), nous avons pu montrer qu'il n'y avait pas besoin de recourir à un instrument symbolique pour développer une stratégie à base d'un diagnostic de régime de fonctionnement de la machine. On avait affaire alors à des machines automatiques à commande manuelle, qui n'étaient pas des MOCN et ne comportaient ni clavier ni écran. Comment procédaient les opérateurs qui s'appuyaient sur un diagnostic? Ils observaient attentivement le mouvement de la machine, en particulier celui qui se produit juste au moment de la commutation. Ils en inféraient la valeur (non quantifiée) des principaux paramètres présents sur la courbe d'une MOCN, ce qui leur permettait d'évaluer le bourrage et ainsi de procéder à un diagnostic de situation. Il est sûr que la disposition de la courbe des pressions rend le diagnostic beaucoup plus facile et précis. Mais il ne faudrait pas confondre l'instrument et le concept.

Nous avons pu observer que, à part deux exceptions (Charles et Lucien), les stratégies de tous les autres régleurs combinaient un pilotage par la courbe et un pilotage par les défauts, même si les différences étaient importantes entre eux, en fonction de leur niveau de compétence. Cela amène à penser que les stratégies mobilisées reposent sur deux sources: une source qui provient directement des résultats des opérations en cours; et une source issue de la représentation conceptuelle que se sont construite les régleurs. La conduite de l'action en effet, son organisation interne ne peuvent négliger ni l'information fournie par les données, ni l'information fournie par les représentations. Une stratégie qui reposerait uniquement sur une représentation conceptuelle de la situation, en reléguant à la fin du processus de résolution de problèmes le contrôle par les données, risque d'être prise au dépourvu dès qu'elle rencontre une situation qui n'a pas été prévue par la représentation abstraite qu'elle a construite. À l'inverse, une stratégie qui reposerait uniquement sur la prise en compte des données factuelles risque de ne pas savoir prendre en compte les détours nécessaires de l'action, qui éloignent provisoirement du but, mais qui permettent de gérer les situations systémiques; elle risque de multiplier à l'infini les épisodes d'essais et erreurs et le recours à des heuristiques générales, sollicitées dès qu'on a le sentiment d'être dans une impasse. Nous pourrions dire que l'organisation de l'activité, et son apprentissage, progressent sur deux pieds, l'empirique et le conceptuel, qui sont tout aussi nécessaires l'un que l'autre. Mais ces deux pieds ne sont pas à égalité : à mesure que le sujet progresse dans son apprentissage, il se construit une représentation fonctionnelle, ou un modèle opératif, à qui il délègue de plus en plus la conduite de l'action. Mais cette délégation demeure sous le contrôle des faits. C'est pourquoi la stratégie la plus aboutie n'est pas la stratégie C, mais la stratégie CD intégrée. On peut penser que cette articulation de deux sources, empirique et conceptuelle, pour fonder une stratégie, est un résultat qui va au-delà de la seule conduite de presses à injecter en plasturgie: la conduite et le réglage de systèmes techniques automatisés, avec ou sans instruments, ne peut pas faire abstraction de la prise en compte des résultats immédiats des opérations, mais ne peut pas se passer d'un modèle opératif, de nature conceptuelle, qui permet une orientation de l'activité.

\section{3.- Niveaux de conceptualisation}

La didactique professionnelle se donne pour objectif d'analyser les apprentissages professionnels en focalisant sur l'activité de conceptualisation. Les résultats obtenus dans cette recherche sont intéressants de ce point de vue. On peut pour le comprendre s'inspirer d'une distinction qu'on trouve chez Cassirer $(1910,1977)$ : ce philosophe distingue ce qu'il appelle les « concepts substances » et les « concepts fonctions ». Un concept substance permet d'identifier une propriété appartenant à un objet. De ce fait, il permet d'identifier une relation stable entre deux objets caractérisés chacun par une propriété. Il permet donc de définir une relation de causalité entre un objet $\mathrm{A}$ (caractérisé par une propriété $\mathrm{P}$ ) et un objet $\mathrm{B}$ (caractérisé par une propriété $\mathrm{P}$ '). Mais dans le cadre des concepts substances, si la relation n'est pas absente, elle est très réduite. En revanche, un concept fonction porte sur un système, caractérisé par un ensemble de relations entre des entités multiples. La focalisation porte, non sur les entités, mais sur les relations entre les entités, le résultat d'ensemble étant fonction de la détermination de chacun des facteurs. Les lois qu'on trouve en physique reposent sur des concepts fonctions. 
Comparons maintenant la stratégie D de Lucien et la stratégie CD de Jacques. Le modèle opératif de Lucien ne manque pas de conceptualisation. La preuve en est qu'il résout tous les problèmes en raison de la règle de fin de problème (R3) qu'il s'est donnée, avec un minimum d'erreurs et très peu de recours à des heuristiques générales. Le modèle opératif de Lucien repose sur un solide substrat de connaissances. Mais toutes ces connaissances relèvent de concepts substances: il s'agit toujours de relier un défaut à une cause. Certes il peut y avoir plusieurs causes possibles pour un défaut et le régleur va les essayer les unes après les autres en fonction de leur degré de probabilité. Mais on ne peut pas dire que ce répertoire de relations binaires forme système. C'est pourquoi Lucien n'a pas les moyens de traiter les compensations. En fait, Lucien s'est construit une stratégie qui lui permet de faire l'économie du diagnostic du régime de fonctionnement de la machine. Le répertoire de règles d'actions qu'il a élaboré est suffisamment précis pour qu'il dispose d'une stratégie efficace, du moins pour les problèmes simples. C'est la raison pour laquelle, sur simulation, Lucien a tout simplement transposé la règle de fin de problème (R3) qu'il utilise dans l'atelier: ignorer les compensations et se contenter d'éliminer les défauts inacceptables.

Jacques, au contraire, a un modèle opératif à base de concepts fonctions. Dès qu'il soupçonne un problème complexe, il cherche à identifier l'ensemble des paramètres impliqués dans un résultat qui peut représenter un défaut ou plusieurs. Dans son esprit la température ou la contre-pression peuvent avoir un effet indirect sur un manque ou une bavure causés par un défaut ou un excès de pression de commutation. L'importance accordée dans cette stratégie aux situations de compensation correspond au fait que ces situations sont caractéristiques de la dimension systémique de la situation. Les situations de compensation obligent à mobiliser des concepts pragmatiques, principalement bourrage et retrait, qui sont des concepts fonctions, dans la mesure où ils permettent de faire un diagnostic du régime de fonctionnement de la machine. Comme nous l'avons vu plus haut, les situations de compensation se distribuent en trois groupes: 1/ il y a les compensations entre le processus d'injection et le processus de maintien, par exemple quand un déficit de matière injectée est compensé, pendant le maintien, par une forte pression de maintien qui correspond à une $2^{\mathrm{e}}$ injection. Le concept pragmatique de bourrage est représentatif de cette situation. 2/ Quand il y a sur les produits des problèmes de cotes ou de serrage, le couple bourrage - retrait représente un $2^{\mathrm{e}}$ type de compensation. Le bourrage relève du processus de conduite de la machine; le retrait est une propriété physique des plastiques, correspondant au retrait de la matière au moment de sa solidification. 3/ Enfin, dernier type de compensation, le processus d'injection - maintien se fait sous certaines conditions générales, soit de température, soit d'état d'usure des pièces de la machine: une température inadaptée ou une buse matée viennent modifier le résultat des réglages. Ces trois types de compensations sont plus ou moins faciles à être représentés par des concepts fonctions. Le premier type de compensation, exprimé par le concept pragmatique de bourrage, est relativement facile d'accès: la plupart des régleurs qui mobilisent une stratégie CD éclatée la maîtrisent. Les deux autres types de compensations sont beaucoup plus difficiles à conceptualiser: il faut une stratégie $\mathrm{CD}$ intégrée (ou sa variante $\mathrm{C}$ ) pour en venir à bout.

La conclusion que nous pouvons tirer de cette discussion est qu'on peut estimer que les régleurs qui réussissent à passer d'une forme de stratégie à une autre procèdent à un véritable saut dans leur conceptualisation. Reprenons les exemples de Daniel et Christophe, ces deux jeunes régleurs qui progressent de façon décisive en cours d'exercice. Nous pouvons supposer que chaque moment de progression correspond au fait de surmonter une difficulté de conceptualisation. Ainsi le passage d'une stratégie pseudo CD chez Daniel à une stratégie CD éclatée correspond au passage d'une représentation en termes de concepts substances à une représentation en termes de concepts fonctions, mais portant uniquement sur le bourrage. Chez Christophe ou Daniel, le passage d'une stratégie CD éclatée à une stratégie $\mathrm{CD}$ intégrée correspond à une généralisation de la représentation en termes de concepts fonctions, qui permet d'englober les trois formes de situations compensées. Certes entre les deux séries de problèmes qu'ils ont traités ces deux régleurs ont pu bénéficier d'une formation sur simulateur qui les mettait en posture d'analyse ${ }^{9}$. On peut penser que ce facteur explique pour une

9. Cette formation n'est pas présentée dans cet article. On peut se reporter à Pastré (2004) pour voir comme elle a été conçue et quels en furent les résultats. 
bonne part l'importance de leur progression d'apprentissage. Mais d'autres régleurs, qui n'ont pas pu bénéficier de cette formation et qui ont pu faire les deux séries d'exercices, ont effectué aussi des progrès dans leur conceptualisation. Ces progrès sont certes beaucoup moins importants, mais ils sont réels. On peut penser que la formation accentue et systématise un processus qui est à l'œuvre dans l'apprentissage informel qui se produit en cours d'activité.

Au final, nous pouvons dire que Lucien comme Jacques, Daniel ou Christophe conceptualisent la situation chacun à leur manière pour orienter et guider leur activité. Mais dans la conceptualisation il y a un moment où s'opère un saut qualitatif. Le recours à la distinction de Cassirer entre concept substance et concept fonction est une manière de penser ce moment de rupture.

\section{Conclusion}

Une bonne manière d'évaluer la compétence de professionnels est de les confronter à des situations critiques, des situations qui posent problème pour eux, en ce sens qu'ils ne possèdent pas, a priori, de procédure pour résoudre le problème qui se pose à eux. La recherche présentée correspond à ce cas de figure: il s'agit de voir comment des opérateurs professionnels procèdent à la résolution des problèmes qu'ils rencontrent dans leur travail. Le fait d'avoir choisi d'utiliser un simulateur a entraîné une conséquence: nous nous sommes situés d'emblée dans un dispositif d'apprentissage et, en un sens, de formation. Certes ce sont des professionnels à qui nous avons affaire, non des novices apprenant les rudiments du métier. Mais ces professionnels sont confrontés aux problèmes les plus difficiles qu'ils sont susceptibles de rencontrer dans leur travail. Et, du coup, ils apprennent en faisant. Autrement dit, en proposant à des professionnels des problèmes complexes extraits de leur travail, non seulement on peut évaluer le niveau de leur compétence professionnelle, mais on les met aussi en posture d'apprentissage: aussitôt qu'il y a exercice de l'activité, il y a une forte probabilité d'apprentissage, d'apprentissage par la résolution des problèmes abordés.

La classe de situations dans laquelle se situe l'analyse que nous avons faite est celle de la supervision de systèmes techniques. Nous ne ferons pas de différences entre conduite, réglage et supervision: dès qu'on a affaire à une machine ou un système technique automatisé, la principale fonction d'un opérateur n'est pas de produire, mais de surveiller la production en intervenant dès que se produit un dysfonctionnement. C'est pourquoi on peut dire que dans le cas de presses à injecter à commande numérique le véritable conducteur est le régleur: sa fonction est de superviser le processus de fabrication. Mais la supervision de ces systèmes techniques automatisés ne constitue pas une classe homogène. Nous pouvons distinguer trois grandes catégories : 1) la correction des dysfonctionnements peut être envisagée comme une suite de situations où un dysfonctionnement est généré par une cause unique. Il peut certes y avoir plusieurs causes possibles pour un même dysfonctionnement, avec pour chacune une certaine probabilité d'apparition. Mais, dans ce premier cas, pour toute situation donnée, la cause du dysfonctionnement est unique. En conséquence, il suffit de posséder le répertoire complet des règles d'action pour être capable de traiter tous les problèmes. On peut se passer d'un diagnostic de situation. Cela ne veut pas dire que les opérateurs n'ont pas conceptualisé la situation. Mais cela signifie qu'il n'est pas possible de faire la différence entre appliquer une procédure et conceptualiser cette dernière. 2) La deuxième catégorie correspond aux situations systémiques: un dysfonctionnement est le produit de plusieurs causes, qui agissent de concert, avec des phénomènes d'accentuation, d'atténuation ou d'inhibition. De nombreuses situations de conduite de systèmes industriels, comme par exemple la conduite d'un train de laminage en sidérurgie (Guibert, 2005), appartiennent à cette catégorie. La conduite de presses à injecter a la particularité de combiner les deux catégories ci-dessus: elle comporte des problèmes simples, avec une relation « une cause - un défaut ». Elle comporte des problèmes complexes, du type «multi-causes - multi-effets ». 3) la troisième catégorie correspond à la conduite de systèmes techniques dynamiques, comme le pilotage d'avions ou la conduite de centrales nucléaires: non seulement on y trouve des configurations systémiques entre variables, mais la dimension temporelle est tellement importante que chaque situation 
doit y être analysée en tenant compte de sa singularité, au point qu'on ne peut pas en faire une analyse en termes de résolution de problèmes par transformation d'états: il faut tenir compte de la dynamique du système, qui ne se réduit pas au résultat des opérations effectuées par les acteurs. La conduite de presse à injecter ne relève clairement pas de cette troisième catégorie.

Par contre, la conduite de presses à injecter à commande numérique peut être considérée comme représentative des activités de conduite de systèmes techniques statiques impliquant des problèmes complexes. Ces situations sont très fréquentes dans l'industrie. Dans une perspective de didactique professionnelle, elles sont très intéressantes, car elles mettent bien en relief les processus de conceptualisation mis en œuvre par les opérateurs. Ce travail de conceptualisation, reposant sur des concepts pragmatiques ou des concepts scientifiques pragmatisés, est ce qui permet de comprendre comment ces opérateurs arrivent à maîtriser des problèmes comportant des configurations systémiques entre variables. Nous avons vu que tous les acteurs n'en ont pas le même niveau de maitrise, d'où la mobilisation de stratégies qui ne comportent pas la même puissance de traitement. Et nous avons pu observer un moment significatif: celui où les opérateurs parviennent à construire des concepts-fonctions.

La recherche que nous avons présentée est le résultat d'une collaboration étroite entre chercheurs venant de trois horizons différents: psychologie cognitive, intelligence artificielle, didactique professionnelle. Nous l'avons mentionné en introduction. Il est important de le rappeler en conclusion: nous espérons avoir montré la fécondité d'une telle démarche, qui n'est certes pas facile à mettre en œuvre, mais qui ouvre de véritables horizons théoriques et méthodologiques. Entre la didactique professionnelle, qui cherche à analyser les apprentissages professionnels, et les approches qui visent à modéliser l'activité, soit par les contraintes, soit par les connaissances, la fécondation a été réciproque : la didactique professionnelle a fourni au départ un matériau pour les approches de modélisation de l'activité. Mais en retour les essais de modélisation de l'activité ont permis une analyse beaucoup plus fine des stratégies d'apprentissage.

\section{RÉFÉRENCES}

Bobrow, D-G. (1984) (Ed.). Qualitative reasoning about physical systems. Amsterdam: North Holland.

Cassirer, E. (1910, 1977). Substance et fonction. Eléments pour une théorie du concept. Paris: Editions de Minuit.

Guibert, S. (2005). Accompagner la transmission de savoir-faire au sein d'un métier our innover et maintenir une performance industrielle. In P. Pastré (Ed.), Apprendre par la simulation (pp. 107-130). Toulouse: Octarès Editions.

Hatchuel, A., \& Weil, B. (1992). L'expert et le système. Paris: Economica.

Hoc, J-M. (1990). Les activités de diagnostic. In J.-F. Richard, C. Bonnet, \& R. Ghiglione (Eds.), Le traitement de l'information symbolique. Traité de psychologie cognitive 2 (pp. 158-162). Paris: Dunod.

Labat, J-M., Pastré, P., Parage, P., Futtersack, M., Richard, J-F., \& Sander, E. (2007). Analyser les stratégies de résolution de problèmes en situation naturelle grâce à un simulateur: le cas des régleurs de plasturgie. In INRP (Eds.), Environnements Informatiques pour l'Apprentissage Humain (pp. 497-508). Lausanne.

Newell, A., \& Simon, H-A., (1972). Human Problem Solving. Eglewood Cliffs, N.J: Prentice Hall.

Pastré, P. (1992). Requalification des ouvriers specialises et didactique professionnelle. Education Permanente, 111, 33-54.

Pastré, P. (1994). Evolution des compétences et formation: le cas de régleurs de presses à injecter. Rapport de fin d'étude d'une recherche financée par le Ministère de la Recherche et de l'Enseignement Supérieur et par l'Agefos-Pme Bourgogne

Pastré, P. (2004). Le rôle des concepts pragmatiques dans la gestion de situations-problèmes : le cas de régleurs en plasturgie. In R. Samurçay, \& P. Pastré (Eds.), Recherches en didactique professionnelle (pp. 17-47). Toulouse: Octarès Editions. 
Pastré, P. (2005). Apprendre par la résolution de problèmes : le rôle de la simulation. In P. Pastré (Ed.), Apprendre par la simulation (pp. 17-40). Toulouse: Octarès Editions.

Pastré, P. (2008). La didactique professionnelle: origines, fondements, perspectives. Travail et apprentissages, 1, 9-21.

Richard, J-F. (1999). Comportements, buts et représentations. Psychologie Française, 44, 75-90.

Richard, J-F., Pastré, P., Parage, P., Sander, E., Futtersack, M., \& Labat J-M. (sous presse). Analyse des stratégies de correction de défauts en plasturgie à l'aide d'un modèle de résolution de problèmes à base de contraintes. Le Travail Humain.

Richard, J-F., Poitrenaud, S., \& Tijus, C. (1993). Problem solving restructuration: Elimination of implicit constraints. Cognitive Science, 17, 497-529.

Samurçay R., \& Rogalski, J. (1992). Formation aux activités de gestion d'environnements dynamiques: concepts et méthodes. Education Permanente, 111, 227-241.

Vergnaud, G. (1996). Au fond de l'action, la conceptualisation. In J.-M. Barbier (Ed.), Savoirs théoriques et savoirs d'action (pp. 275-291). Paris: PUF. 


\section{Annexe 1 \\ Règles permettant de modéliser l'activité}

\section{1.- Règles de gestion de la tâche $(G)$}

On a à gauche la stratégie à laquelle elles appartiennent: $\mathrm{C}$ pour courbe, $\mathrm{D}$ pour défaut, $\mathrm{CD}$ pour courbe et défauts. Au milieu on trouve les conditions. La condition commune à toutes les règles de la case est en gras. À droite, la décision

\begin{tabular}{|c|c|c|}
\hline Stratégie & Conditions & Action \\
\hline & \multicolumn{2}{|l|}{ Il n'y a pas encore eu de réglage } \\
\hline $\mathrm{C}$ & La courbe est absente et lisible & Demander la courbe \\
\hline $\mathrm{CD}$ & $\begin{array}{l}\text { La courbe est absente et lisible, mais si le défaut a } \\
\text { une cause unique }\end{array}$ & Demander la courbe ou faire un réglage \\
\hline C-CD & La courbe est présente & Faire un réglage \\
\hline $\mathrm{CD}$ & $\begin{array}{l}\text { La courbe est présente, mais s'il y a } \\
\text { incompatibilité entre la courbe et le défaut }\end{array}$ & Redemander courbe ou défaut \\
\hline \multirow[t]{2}{*}{ C-CD-D } & La courbe n'est pas lisible & Faire un réglage \\
\hline & \multicolumn{2}{|c|}{ Il y a eu un réglage sur paramètre de la courbe lisible } \\
\hline C-CD & Ni les défauts ni la courbe n'ont été demandés & Demander la courbe \\
\hline $\mathrm{CD}$ & Ni les défauts ni la courbe n'ont été demandés & Demander la courbe ou le défaut \\
\hline $\mathrm{C}-\mathrm{CD}$ & $\begin{array}{l}\text { La courbe est normale, le défaut n'a pas été } \\
\text { demandé }\end{array}$ & Demander le défaut \\
\hline $\mathrm{C}$ & La courbe n'est pas normale & Faire un réglage ou demander le défaut \\
\hline $\mathrm{CD}$ & La courbe n'est pas normale & Faire un réglage ou demander le défaut \\
\hline $\mathrm{CD}$ & Il y a encore des défauts & Faire un réglage ou demander la courbe \\
\hline \multirow[t]{2}{*}{$\mathrm{CD}$} & Il n'y a plus de défauts & Demander la courbe \\
\hline & \multicolumn{2}{|l|}{ Il y a eu un réglage sur paramètre courbe non lisible } \\
\hline C-CD-D & Ni les défauts ni la courbe n'ont été demandés & Demander le défaut \\
\hline C-D & Il y a encore des défauts & Faire un réglage \\
\hline \multirow[t]{2}{*}{$\mathrm{CD}$} & $\begin{array}{l}\text { Il y a encore un défaut, le défaut est inchangé ou } \\
\text { aggravé }\end{array}$ & Faire un réglage ou demander la courbe \\
\hline & \multicolumn{2}{|l|}{ Il y a eu un réglage sur paramètre hors courbe } \\
\hline C-CD-D & Il n'y a pas eu de demande de défauts & Demander le défaut \\
\hline C-D & Il y a encore des défauts & Faire un réglage \\
\hline \multirow[t]{2}{*}{$\mathrm{CD}$} & $\begin{array}{l}\text { Il y a encore un défaut, le défaut est inchangé ou } \\
\text { aggravé }\end{array}$ & Faire un réglage ou demander la courbe \\
\hline & \multicolumn{2}{|c|}{ Il y a eu un réglage et au moins une demande de courbe et une demande de défaut } \\
\hline $\mathrm{C}$ & $\begin{array}{l}\text { Si la courbe n'est pas normale ou s'il y a des } \\
\text { défauts }\end{array}$ & Faire un réglage \\
\hline $\mathrm{CD}$ & $\begin{array}{l}\text { Soit la courbe est normale et il y a encore des } \\
\text { défauts, soit la courbe n'est pas normale et il n'y a } \\
\text { plus de défauts }\end{array}$ & $\begin{array}{l}\text { Faire un réglage ou demander la courbe ou le défaut } \\
\text { (selon ce qui a été fait auparavant) }\end{array}$ \\
\hline $\mathrm{CD}$ & $\begin{array}{l}\text { Si la courbe est anormale et qu'il y a encore des } \\
\text { défauts et que les paramètres défectueux de la } \\
\text { courbe ne correspondent pas aux causes connues } \\
\text { des défauts }\end{array}$ & $\begin{array}{l}\text { Faire un réglage ou demander la courbe ou le défaut } \\
\text { (selon ce qui a été fait auparavant) }\end{array}$ \\
\hline
\end{tabular}

\section{2.- Règles de réglage (liées aux connaissances du domaine)}

Plutôt que de faire la liste de toutes les règles de réglage, on fournit les connaissances du domaine (relation entre paramètres et défauts): les règles peuvent en être déduites. Par exemple: si Bavure, alors diminuer $\mathrm{PC}$, ou $\mathrm{T}^{\circ}$, ou $\mathrm{Cp}$ ou augmenter TID. 


\begin{tabular}{|c|c|c|c|c|}
\hline Défauts & Actions & Causes & Défauts & Actions \\
\hline Manque & \begin{tabular}{|l}
$\mathrm{PC}(+)$ \\
$\mathrm{T}^{\circ}(+)$ \\
$\mathrm{CP}(+)$ \\
$\mathrm{TID}(-)$ \\
Buse
\end{tabular} & Quantité de matière injectée & Bavure & $\begin{array}{l}\mathrm{PC}(-) \\
\mathrm{T}^{\circ}(-) \\
\mathrm{CP}(-) \\
\mathrm{TID}(+)\end{array}$ \\
\hline Retassure & $\begin{array}{l}\mathrm{P} 2(+) \\
\mathrm{T} 2(+)\end{array}$ & Compensation du retrait & Dépression & $\begin{array}{l}\text { P2 (-) } \\
\text { T2 (-) }\end{array}$ \\
\hline Striage & TID (-) & Vitesse d'injection & Brûlure & TID (+) \\
\hline Serrage faible & $\begin{array}{l}\mathrm{T} 2(+) \\
\mathrm{T} 3(+) \\
\mathrm{P} 2(-) \\
\mathrm{T}^{\circ}(-)\end{array}$ & $\begin{array}{l}\text { Compensation du retrait } \\
\text { Echanges thermiques }\end{array}$ & Serrage fort & $\begin{array}{l}\mathrm{T} 2(-) \\
\mathrm{T} 3(-) \\
\mathrm{P} 2(+) \\
\mathrm{T}^{\circ}(+)\end{array}$ \\
\hline
\end{tabular}

\section{3.- Règles de réglage liées à l'utilisation d'heuristiques}

a- Heuristiques liées à la mémorisation:

- Ne pas faire le même réglage qu'à l'essai précédent

- Ne pas répéter un réglage qui a déjà été fait sur le problème, sauf si la répétition a lieu immédiatement (pour changer de 2 valeurs le paramètre) ou après être revenu en arrière (parce que le réglage était interdit ou parce qu'il y avait eu une aggravation du défaut), pour mettre le paramètre à une valeur différente.

- Ne pas agir deux fois (de suite ou non) dans les 2 essais précédents du problème sur le même paramètre sauf si l'action est interdite ou si c'est pour revenir en arrière, ou si c'est après être revenu en arrière afin de mettre le paramètre à une valeur différente.

b- Heuristiques liées au feedback

- Si le réglage est interdit, faire le réglage inverse immédiatement ou le coup d'après.

- Si le dernier réglage est valide, s'il y a eu une demande de défaut après l'avant-dernier réglage, et s'il y a déteriorisation du défaut, alors faire le réglage inverse.

- Si le dernier réglage est valide, s'il y a eu une demande défaut après l'avant-dernier réglage et si le défaut est aggravé ou inchangé, alors faire le réglage inverse.

\section{4.- Règles fixant les conditions d'arrêt du problème :}

- R3: On arrête s'il n'y a plus de défauts, sauf pour le serrage pour lequel un écart léger en plus ou en moins est toléré.

- R2: On arrête s'il n'y a plus du tout de défaut, mais on ne tient pas compte de la courbe.

- R1: On arrête s'il n'y a plus du tout de défaut et si les paramètres de la courbe sont à leur valeur normale. 


\section{Annexe 2}

\section{Un exemple d'analyse de la solution d'un problème en termes de règles}

\begin{tabular}{|c|c|c|c|c|c|c|c|c|c|c|}
\hline \multicolumn{4}{|c|}{ Défauts } & \multicolumn{5}{|c|}{ Paramètres courbe } & \multirow[t]{2}{*}{ Règles } & \multirow[t]{2}{*}{ Actions } \\
\hline $\mathrm{B} / \mathrm{M}$ & $\mathrm{Br} / \mathrm{St}$ & $\mathrm{Re} / \mathrm{C}$ & $\mathrm{Se}$ & $\mathrm{Ti}$ & $\mathrm{Pc}$ & $\mathrm{Cp}$ & $\mathrm{P} 2$ & $\mathrm{~T} 2$ & & \\
\hline \multirow[t]{5}{*}{$\mathrm{N}$} & $\mathrm{N}$ & $\mathrm{N}$ & S1 & & & & & & $\begin{array}{l}\text { T1 : s'il n'y a pas encore eu de réglage, il y a les défauts, } \\
\text { la courbe est lisible, il n'y a pas de courbe, demander la } \\
\text { courbe }\end{array}$ & $1:$ Courbe \\
\hline & & & & 0 & -1 & 0 & 1 & 1 & $\begin{array}{l}\text { T2 : s'il n'y a pas encore eu de réglage, il y a les défauts, } \\
\text { la courbe est lisible, la courbe est présente, faire un } \\
\text { réglage }\end{array}$ & 2 : Réglage \\
\hline & & & & 0 & -1 & 0 & 1 & 1 & $\begin{array}{l}\text { R1 : Causes connues prioritaires du défaut : } \mathrm{P} 2 \text { et/ou T2 } \\
\text { trop fortes. But : diminuer P2 ou T2. Choix du protocole } \\
: \text { diminuer P2 }\end{array}$ & $3:$ P2- \\
\hline & & & & 0 & -1 & 0 & 0 & 1 & $\begin{array}{l}\text { T3 : après un réglage valide qui n'est pas un retour en } \\
\text { arrière, demander de l'information T4: Si l'on doit } \\
\text { demander de l'information, si le réglage porte sur un } \\
\text { paramètre de la courbe lisible, demander la courbe ou le } \\
\text { défaut }\end{array}$ & 4: Courbe \\
\hline & & & & 0 & -1 & 0 & 0 & 1 & $\begin{array}{l}\text { T5: le réglage porte sur un paramètre de la courbe } \\
\text { lisible, il y a la courbe, pas les défauts, demander les } \\
\text { défauts ou faire un réglage }\end{array}$ & 5 : Défauts \\
\hline \multirow[t]{6}{*}{ M1 } & $\mathrm{N}$ & $\mathrm{N}$ & & & & & & & $\begin{array}{l}\text { T6: s'il y a eu un réglage sur un paramètre de la courbe, } \\
\text { la courbe est incorrecte, il y a des défauts, faire un } \\
\text { réglage }\end{array}$ & 6 : Réglage \\
\hline & & & & & & & & & $\begin{array}{l}\text { T7 : S'il y a eu un réglage valide et une détérioration du } \\
\text { défaut, annuler le réglage, donc augmenter P2 }\end{array}$ & $7: \mathrm{P} 2+$ \\
\hline & & & & 0 & -1 & 0 & 0 & 1 & $\begin{array}{l}\text { T8: S'il y a eu un réglage valide, mais si c'est un retour } \\
\text { en arrière, faire un nouveau réglage (ou demander de } \\
\text { l'information) }\end{array}$ & 8 : Réglage \\
\hline & & & & & & & & & $\begin{array}{l}\text { R1 : même état qu'en } 3: \text { les causes du défaut sont } \\
\text { toujours P2 et/ou T2 trop fortes, P2 a été essayée, } \\
\text { diminuer T2 }\end{array}$ & $9: \mathrm{T} 2-$ \\
\hline & & & & & & & & & $\begin{array}{l}\text { T3: Après un réglage valide qui n'est pas un retour } \\
\text { en arrière, demander de l'informationT4 : si l'on doit } \\
\text { demander de l'information, si le réglage porte sur un } \\
\text { paramètre de la courbe lisible, demander la courbe ou le } \\
\text { défaut }\end{array}$ & 10 : Courbe \\
\hline & & & & 0 & -1 & 0 & 1 & 1 & $\begin{array}{l}\text { T5: Le réglage porte sur un paramètre de la courbe } \\
\text { lisible, il y a la courbe, pas les défauts, demander les } \\
\text { défauts ou faire un réglage }\end{array}$ & 11 : Défauts \\
\hline \multirow[t]{6}{*}{$\mathrm{N}$} & $\mathrm{N}$ & $\mathrm{N}$ & S1 & & & & & & $\begin{array}{l}\text { T9: S'il y a eu un réglage valide sur un paramètre de la } \\
\text { courbe, il y a des défauts, faire un réglage ou demander } \\
\text { la courbe }\end{array}$ & 12 : Courbe \\
\hline & & & & 0 & -1 & 0 & 1 & 0 & $\begin{array}{l}\text { T9 : S'il y a eu un réglage valide sur un paramètre de } \\
\text { la courbe, il y a la courbe, il y a des défauts, faire un } \\
\text { réglage }\end{array}$ & 13 : Réglage \\
\hline & & & & & & & & & $\begin{array}{l}\mathrm{R} 2: \text { Actions compatibles avec le but et la courbe : } \mathrm{Pc}+/- \text {, } \\
\mathrm{Cp}+/-, \mathrm{P} 2-. \text { Mais } \mathrm{P} 2 \text { a été tenté récemment. Restent } \mathrm{Pc}+ \\
\mathrm{Pc}-, \mathrm{Cp}+, \mathrm{Cp}-\end{array}$ & $\begin{array}{l}14: \text { PC- } \\
\text { Interdit }\end{array}$ \\
\hline & & & & & & & & & H1: Si un réglage est interdit, faire le réglage inverse & $15: \mathrm{Pc}+$ \\
\hline & & & & & & & & & $\begin{array}{l}\text { T8 : S'il y a eu un réglage valide, mais si c'est un retour } \\
\text { en arrière, faire un nouveau réglage ou demander de } \\
\text { l'information T4 : si l'on doit demander de l'information, } \\
\text { si le réglage porte sur un paramètre de la courbe lisible, } \\
\text { demander la courbe ou le défaut }\end{array}$ & 16 : Courbe \\
\hline & & & & 0 & 0 & 0 & 1 & 0 & $\begin{array}{l}\text { T5: Le réglage porte sur un paramètre de la courbe } \\
\text { lisible, il y a la courbe, pas les défauts, demander les } \\
\text { défauts ou faire un réglage }\end{array}$ & 17 : Défauts \\
\hline $\mathrm{N}$ & Cas & $\mathrm{N}$ & $\mathrm{S} 1$ & & & & & & $\begin{array}{l}\mathrm{T} 7: \mathrm{S} \text { 'il y a eu un réglage valide et une détérioration du } \\
\text { défaut, annuler le réglage, donc diminuer Pc }\end{array}$ & $18:$ Pc- \\
\hline
\end{tabular}




\begin{tabular}{|c|c|c|c|c|c|c|c|c|c|c|}
\hline & & & & & & & & & $\begin{array}{l}\text { T8 : S'il y a eu un réglage valide, mais si c'est un retour } \\
\text { en arrière, faire un nouveau réglage (ou demander de } \\
\text { l'information) }\end{array}$ & 19: Réglage \\
\hline & & & & & & & & & $\begin{array}{l}\text { R3 : la seule action compatible avec la réduction du } \\
\text { défaut S1 est P2-. P2- n'a pas été tenté récemment : faire } \\
\text { P2- }\end{array}$ & 20: P2- \\
\hline & & & & & & & & & $\begin{array}{l}\text { T3 : Après un réglage valide qui n'est pas un retour } \\
\text { en arrière, demander de l'information T4 : si l'on doit } \\
\text { demander de l'information, si le réglage porte sur un } \\
\text { paramètre de la courbe lisible, demander la courbe ou le } \\
\text { défaut }\end{array}$ & $21:$ Courbe \\
\hline & & & & 0 & -1 & 0 & 0 & 0 & $\begin{array}{l}\text { T5 : Le réglage porte sur un paramètre de la courbe } \\
\text { lisible, il y a la courbe, pas les défauts, demander les } \\
\text { défauts ou faire un réglage }\end{array}$ & 22 : Réglage \\
\hline & & & & & & & & & $\begin{array}{l}\mathrm{R} 4 \text { : Actions compatibles avec la courbe : } \mathrm{Pc}+/-, \mathrm{Cp}+/- \text { : } \\
\text { sont possibles } \mathrm{Pc}+, \mathrm{Pc}-, \mathrm{Cp}+, \mathrm{Cp}-\end{array}$ & $23: \mathrm{Pc}+$ \\
\hline & & & & & & & & & $\begin{array}{l}\text { T3 : Après un réglage valide qui n'est pas un retour } \\
\text { en arrière, demander de l'information T4 : si l'on doit } \\
\text { demander de l'information, si le réglage porte sur un } \\
\text { paramètre de la courbe lisible, demander la courbe ou le } \\
\text { défaut }\end{array}$ & 24 : Défauts \\
\hline $\mathrm{N}$ & $\mathrm{N}$ & $\mathrm{N}$ & $\mathrm{N}$ & & & & & & $\begin{array}{l}\text { Règle d'arrêt R1 : s'il n'y a plus de défaut et si la courbe } \\
\text { est normale, le problème est terminé. }\end{array}$ & \\
\hline & & & & & & & & & & \\
\hline
\end{tabular}

\section{Légende:}

1. Colonne 1 (Défauts):

$$
\begin{aligned}
& \mathrm{B} / \mathrm{M}=\text { bavures/manques } \mathrm{Br} / \mathrm{St}=\text { brûlure/striage } \\
& \mathrm{Re} / \mathrm{C}=\text { retassures/risques de cassure } \\
& \mathrm{Se}=\text { serrage } \\
& \mathrm{S} 1=\text { serrage légèrement fort } \\
& \mathrm{M} 1=\text { manque léger } \\
& \mathrm{Cas}=\text { risque de cassure } \\
& \mathrm{N}=\text { normal }
\end{aligned}
$$

2. Colonne 2 (valeurs des paramètres sur la courbe)

$\mathrm{Ti}=$ temps d'injection dynamique

$\mathrm{Pc}=$ pression de commutation

$\mathrm{Cp}=$ contre-pression

$\mathrm{P} 2=$ pression de maintien

$\mathrm{T} 2=$ temps de maintien

3 . Colonne 3 (règles)

$\mathrm{T} 1 \ldots=$ règles de gestion de la tâche

$\mathrm{R} 1 \ldots=$ règles de réglage à partir des connaissances du domaine

$\mathrm{H} 1$ = règles de réglage à partir d'heuristique.

\section{Commentaire}

Cette résolution de problème relève de la stratégie $\mathrm{CD}$ éclatée. En effet:

- les prises d'information coordonnent courbe et défauts (exemples : 4-5; 10-11-12)

— on voit à l'œuvre la règle de la situation dégradée, caractéristique de la stratégie CD éclatée (exemple: item 7).

- Le niveau de connaissances est correct pour P2, T2; insuffisant pour PC (item 14).

\section{RÉSUMÉ}

Ce texte est issu d'une recherche sur la résolution de problèmes professionnels qui a été faite par une équipe regroupant des chercheurs venant de trois horizons différents : psychologie cognitive, intelligence artificielle, didactique professionnelle. On a utilisé le matériau recueilli en 1992-95 par Pierre Pastré et qui portait sur l'activité de régleurs procédant à la correction de défauts sur des objets plastiques produits par des presses à injecter; cette résolution de problèmes professionnels s'est faite sur simulateur. L'objectif de la recherche était de modéliser l'activité des régleurs à partir de deux approches : une approche par les contraintes (Richard), une approche par les connaissances (Labat et al.). Les résultats de ces modélisations ont permis 
en retour une analyse plus fine des stratégies mobilisées par les régleurs. C'est cette analyse des stratégies des régleurs, dans leur organisation et dans leurs évolutions, qui est présentée dans ce papier. L'analyse y est faite dans une perspective de conceptualisation dans l'action. Deux résultats principaux apparaissent: la grande majorité des stratégies des régleurs combinent une approche orientée empirique (conduite par les défauts) et une approche orientée symbolique (conduite par la courbe des pressions), avec une évolution où la part d'approche symbolique prend de plus en plus d'importance, mais sans qu'il y ait disparition de l'approche empirique. Par ailleurs, le moment crucial dans l'évolution des stratégies se situe au moment où les régleurs passent d'une conceptualisation de type linéaire (un défaut - une cause) à une conceptualisation de type systémique (prise en compte des compensations).

\section{MoTS CLÉS}

Simulation, modélisation de l'activité, résolution de problèmes, didactique professionnelle, conceptualisation dans l'action.

\section{RESUMEN}

La resolución de problemas profesionales en simulador. Este texto es el resultado de una investigación sobre los problemas profesionales, realizada por un equipo que agrupa a investigadores que provienen de tres horizontes diferentes: psicología cognitiva, inteligencia artificial, didáctica profesional. Se ha utilizado el material recabado en 1992-95 por Pierre Pastré que trataba de la actividad de los operadores que ajustan las prensas inyectoras de objetos de plástico y que corrigen sus defectos; esta resolución de problemas profesionales se realizo en un simulador. El objetivo de la investigación fue modelizar la actividad de los operadores en cuestión a partir de dos enfoques: el primero se basa en las exigencias (Richard) y el segundo, en los conocimientos (Labat et alii). Los resultados de estas modelizaciones han permitido a su vez realizar un análisis mas detallado de las estrategias movilizadas por los operadores. Este trabajo presenta este análisis de las estrategias de los operadores que realizan los ajustes en las prensas, su organización y sus evoluciones. El análisis se ha realizado desde una perspectiva de conceptualización en la acción, generando dos resultados principales: la gran mayoría de las estrategias de los operadores de ajuste combinan una metodología orientada por lo empírico (guiada por los defectos) y otra orientada por lo simbólico (guiada por la curva de presiones), con una evolución en la que el enfoque simbólico toma cada vez mayor importancia, pero sin que el enfoque empírico desaparezca totalmente. Por otro lado, el momento crucial en la evolución de las estrategias se sitúa en el momento en que los operadores pasan de una conceptualización de tipo lineal (un defecto-una causa) a una conceptualización de tipo sistémico (consideración de las compensaciones).

\section{PaLABRAs Clave}

Simulación, modelización de la actividad, resolución de problemas, didáctica profesional, conceptualización en la acción

\section{RÉFÉRENCEMENT}

Pastré, P., Parage, P., Richard J.-F., Labat, J.-M., \& Futtersack, M. (2009). La résolution de problèmes professionnels sur simulateur. Activités 6 (1), pp. 3-28, http://www.activites.org/ v6n1/v6n1.pdf

Article soumis le 18 mai 2008, accepté pour publication le Accepté le 20 février 2009 
